\title{
MEMORIALES DE GUERRA. RECUERDO Y OLVIDO MÁS ALLÁ DE LAS HUELGAS*
}

\author{
Rose Walker \\ The Courtauld Institute of Art
}

\begin{abstract}
RESUMEN
En este artículo se explora la posibilidad de que construcciones medievales hispanas vinculadas a victorias y derrotas en batalla hayan podido funcionar como "memoriales de guerra". Se tomará al monasterio de Las Huelgas como punto de partida ya que esta fundación fue creada a instancias de Alfonso VIII, un monarca que experimentó ambos extremos durante su reinado: si bien sufrió un doloroso revés en Alarcos (1195), acabaría finalmente recuperando su crédito como líder militar con la victoria de Las Navas de Tolosa (1212), que le granjearía una fama sin parangón en la época. Por su parte, la Orden de Calatrava había sufrido importantes pérdidas en la fallida campaña de Alarcos, y el recuerdo de los mártires caídos en batalla aquella jornada perseguiría durante años a Alfonso VIII. En este sentido, el cuasi privado claustro de Las Claustrillas en Las Huelgas - un espacio performativopudo haber servido como lugar para la penitencia y el olvido, mientras que la iglesia del monasterio burgalés y la fortaleza de Calatrava la Nueva habrían monumentalizado y celebrado la victoria de Las Navas.
\end{abstract}

Palabras clave: Memoriales de guerra; Las Huelgas, Burgos; Alarcos; Las Navas de Tolosa, Calatrava.

\section{ABSTRACT}

This article considers whether structures that are connected with victory and defeat in Medieval Spain can be viewed as 'war memorials'. It will take Las Huelgas as a starting point, since that abbey was the foundation of Alfonso VIII who experienced both extremes. In 1195 his army met catastrophic disaster at Alarcos. In 1212 his reputation as a military leader was restored beyond all imagining by the victory at Las Navas de Tolosa. The Order of Calatrava had been at the centre of the failed campaign at Alarcos, and the martyrdom of their knights continued to haunt Alfonso VIII. The relatively private cloister of Las Claustrillas at Las Huelgas may have been a place of penance and forgetting, a performative space, whereas the church at Las Huelgas and the fortress of Calatrava enshrined and celebrated the memory of victory.

Keywords: War Memorials; Las Huelgas, Burgos; Alarcos; Las Navas de Tolosa, Calatrava

La mayor parte de las obras consagradas al estudio de los memoriales de guerra se deben a investigadores especializados en la Edad Moderna y Contemporánea, y no a medievalistas. ${ }^{1}$ Ciertamente, el material a analizar es demasiado vasto como para ser abordado en conjunto, puesto que incluiría tanto grandes cementerios como monumentos más humildes, con independencia de que éstos se refieran a victorias o derrotas de las que, a veces, poco más queda que un nebuloso recuerdo. Sin embargo, conviene tener presente que construcciones como el Cenotafio de Londres o el Arco de Triunfo en París sirven de aglutinante aún hoy en día para ceremonias conmemorativas a escala nacional, celebradas cada año. A medio camino entre la glorificación del triunfo y el anhelo de paz, estos actos siguen resultando profundamente conmovedores.

\footnotetext{
* Traducción de Rosa María Rodríguez Porto. Los pasajes de la Crónica latina de los reyes de Castilla que en la versión original aparecían en inglés, según la traducción de Joseph F. O'Callaghan (Tempe, 2002), han sido recogidos aquí de acuerdo con la edición castellana de Luis Charlo Brea [N. de la t.].
} 
Una de las características más acusadas de este tipo de monumentos es la atención prestada al soldado anónimo. En 1920, la Tumba del Soldado Desconocido fue erigida en la Abadía de Westminster para recordar "a las grandes multitudes" que murieron durante la Primera Guerra Mundial. Ese mismo año, otra Tumba del Soldado Desconocido vino a unirse a los nombres de quinientos generales franceses inscritos en los muros interiores del Arco de Triunfo parisino. En Alemania, hasta 1931 no se construyó un "Memorial por los caídos en la Guerra" en la Neue Wache, aunque más tarde acabaría siendo lugar de especial significación para el movimiento nazi, de ahí que ahora haya pasado a denominarse oficialmente "Monumento de la República Federal de Alemania a las Víctimas de la Guerra y la Tiranía".

Otro de los aspectos que singularizan a muchos de estos memoriales es su carácter acumulativo, ya que en ellos se recogen los nombres de los muertos en conflictos bélicos desde el siglo XIX en adelante. De alguna manera, estos largos listados parecen aludir también a los muertos de futuras guerras. No es de extrañar, por tanto, que en ocasiones estos monumentos conmemorativos provoquen sentimientos que ni siquiera sus propios creadores podían prever. Así, Mary Carruthers ha llamado la atención sobre el modo en que el Monumento a los Veteranos de Vietnam en Washington D. C. participa de una tradición social establecida, al tiempo que da lugar a respuestas particulares entre los visitantes. La configuración del memorial resulta extremadamente efectiva, al tratarse de un simple muro de "granito negro pulido [con] los nombres de cada uno de los muertos" que se erige entre los monumentos a Washington y a Lincoln. De algún modo, es el ascetismo del conjunto el que estimula a los visitantes a narrar sus historias particulares en el marco de este monumento colectivo. Con todo, estos relatos individuales acaban por convertirse en apostillas al texto del muro del monumento, a través de actos conmemorativos, fotografías, inscripciones de nombres u ofrendas. ${ }^{2}$ Este recuerdo participativo es típico de nuestro tiempo pero, ¿ha de considerarse totalmente anacrónico antes del siglo XIX?, ¿Resta alguna evidencia de un reconocimiento similar de las pérdidas humanas causadas por la guerra durante la Edad Media?

Aparentemente, esta práctica conmemorativa habría tenido su origen en los años que siguieron a la Revolución Francesa. ${ }^{3}$ Aunque los memoriales de finales del siglo XVIII no han sobrevivido, testimonios contemporáneos prueban que fueron erigidos por los jacobinos, partidarios de la Revolución, y que estaban destinados a conmemorar a miembros de ciertas fraternidades. En este sentido, podrían ponerse en relación con algunos precedentes medievales. Por otro lado, es bien sabido que ya desde la Antigüedad se erigían monumentos para conmemorar victorias, como atestiguan los arcos de triunfo y las columnas romanas todavía visibles. En cambio, se antoja mucho más difícil encontrar en ellos alguna muestra tangible de interés por los caídos en combate, y mucho menos por los muertos anónimos. Así, el Ara Pacis de Augusto ofrecía la imagen de un estado fecundo y en paz y, en este sentido, tal vez pretendiese cerrar simbólicamente las heridas abiertas por la guerra civil. No obstante, la memoria del conflicto se habría hecho presente en él sólo como ausencia, por lo que cabría concluir que el objetivo debió de ser pasar página, más que volver la vista hacia el pasado.

Por lo que respecta a la Edad Media, resulta más complicado aún reconocer la existencia de memoriales de guerra, tan inextricablemente unidas estaban entonces la victoria y la derrota a la voluntad divina. ${ }^{4}$ En la concepción medieval, Dios otorgaba la victoria de acuerdo con los méritos de los contendientes, de tal manera que una gran victoria podía afianzar a un monarca en el trono y una derrota, por el contrario, hacerle perder la corona. Más difícil todavía resultaba entender los designios divinos cuando eran dos reyes cristianos los que se enfrentaban. A título de ejemplo, puede señalarse que ciertas fuentes del siglo XII describen la fundación de Battle Abbey en 1070, en el mismo emplazamiento de la batalla de Hastings, como una empresa conmemorativa con la que Guillermo el Conquistador agradecía la victoria que Dios le había concedido, pero también como una forma de expiación por "aquellos que allí perecieron". ${ }^{5}$ De hecho, el altar mayor debía indicar el lugar 
en el que el rey Harald había sido asesinado. Sin embargo, como afirma Elizabeth Hallam, "lo más probable es que el rey considerase todo ello como expresión de su poder y no tanto como gesto de contrición".

Ya en ámbito hispano, cabría mencionar que fue tras el victorioso asedio de la ciudad de Santarém en 1147 cuando Alfonso I de Portugal trajo a monjes cistercienses para fundar Alcobaça (ca. 1153). Pero si bien esta fundación se convertiría en un importante centro religioso del nuevo reino, no puede considerárselo como un memorial de guerra en sentido estricto. ${ }^{6}$ Por el contrario, la intención conmemorativa es más clara en otro monumento del siglo XII: la mezquita de ElHassan de Salé (Rabat), ciudad que fue construida entre 1196 y 1199 para solemnizar la victoria del emir almohade Abu Ya'qub, conocido como Miramamolín, sobre el rey Alfonso VIII de CastiIla en Alarcos. Esta terrible derrota inflingida al monarca castellano le hizo acreedor del título "al-Mansur Billah" (Aquel al que Dios hace victorioso). Diversos relatos contemporáneos aseguran que fueron 30.000 los muertos del lado cristiano y 500 entre los musulmanes, aunque otras fuentes menos fiables elevan estas cifras a 300.000 frente a 20.000 , respectivamente. En cualquier caso, parece fuera de toda duda que las pérdidas por parte de los cristianos superaron con creces a las de sus oponentes. De acuerdo con Al-Himyari, que escribe a principios del siglo $\mathrm{XV}$ basándose en textos anteriores, los almohades también se habrían hecho con un gran botín y con un elevado número de esclavos, a los que habrían obligado a trabajar en la construcción de la mezquita. ${ }^{7}$ Sin embargo, ésta no llegaría a concluirse. Puede que ello se debiese a la muerte de Miramamolín en 1199 o al inesperado y dramático vuelco de la situación en 1212. Con todo, la torre y el extenso espacio reservado para la sala de oración y el patio siguieron dominando el paisaje urbano, en tanto que evocador testimonio del imperio almohade y de la gran mezquita que pudo haber sido.

Los monumentos reseñados parecen carecer de las cualidades hoy asociadas con el término "memorial de guerra". No hay en ellos referencia alguna a los caídos, a los desastres provocados por la guerra o a los soldados anónimos que tomaron parte en la batalla. Para apreciar la particular sensibilidad medieval sobre estos temas es preciso, por el contrario, volver la vista sobre el recuerdo de la derrota. En esta indagación, la batalla de Alarcos servirá de ejemplo una vez más, aunque ahora desde el punto de vista inverso y bien documentado del rey Alfonso VIII de Castilla. El relato más detallado de la derrota se halla en la denominada Crónica latina de los reyes de Castilla. ${ }^{8}$ A partir de esta descripción, puede inferirse que el monarca había subestimado las fuerzas de sus rivales bereberes cuando decidió salir al campo sin aguardar la llegada de su aliado el rey de León. ${ }^{9}$ Sus tropas estaban cansadas y reinaba cierto desconcierto, puesto que Alfonso había ofrecido batalla al emir el día anterior, a lo que éste se había negado. Como consecuencia, resume la crónica que fue aquel "[un] día pródigo en sangre humana, [que] envía moros al tártaro y traslada a los cristianos a los eternos palacios". ${ }^{10}$ También se relata como el rey se lanzó al ataque, si bien sus hombres "le suplicaron que se alejase y preservara su vida ya que el Señor Dios se mostraba airado con el pueblo cristiano". Él así lo hizo, a su pesar, "doliéndose y gimiendo por la gran desgracia que había acontecido". La Orden de Santiago perdió en Alarcos a diecinueve de sus miembros y la mayor parte de la Orden de Calatrava pereció en el campo de batalla. ${ }^{11}$ Los obispos de Ávila, Segovia y Sigüenza también se contaron entre los muertos, así como algunos de los más estrechos colaboradores de Alfonso VIII, incluyendo a su mayordomo Pedro Rodríguez de Guzmán y el yerno de éste, Rodrigo Sánchez, a los que cabría añadir Ordoño García de Roa y sus hermanos. ${ }^{12}$ Sin duda, Alarcos fue una catástrofe política y personal para Alfonso VIII, y el número de víctimas hace de ella, como señala Joseph O'Callaghan, "la derrota más severa sufrida por un monarca cristiano desde que las tropas de Alfonso VI fuesen aplastadas en Zallaqa (Sagrajas) un siglo antes". ${ }^{13}$

El resto del mundo no tardó en comprender la magnitud del desastre. El trovador Fulco de Marsella lamentó la profunda caída de Alarcos (Hueimas no y conosc razo), que traía el recuerdo de la pérdida de Jerusalén en 1187: Qu' el sepulcre perdem premeiramen. ${ }^{14}$ En el poema, se responsabilizaba implícitamente a Alfonso VIII 
del fracaso al presentar batalla sin tener a Dios de su parte, puesto que "cualquier esfuerzo sin Dios es en vano", aunque la crítica no fuese más allá. Ciertamente, Dios debía de estar muy airado con los cristianos para haber permitido semejante derrota. Pero el poema de Fulco era también una llamada a las armas y, dado su origen provenzal, no sería extraño que en él se urgiese la colaboración del rey de Aragón en la ofensiva. Su opinión no era desinteresada, ni carecía de partidarios. En Inglaterra, Matthew Paris escribía en su Chronica Majora que en 1195 "toda la Cristiandad, sumida en la confusión, se había estremecido hasta sus cimientos". ${ }^{15}$ Retrospectivamente, hasta el gran cronista regio, el Arzobispo de Toledo Jiménez de Rada, se permitiría aludir en presencia del rey, después de la victoria de las Navas de Tolosa en 1212, a aquel "deshonor soportado por un tiempo" (oprobrium aliquandiu toleratum). ${ }^{16}$

La lectura de las fuentes coetáneas sugiere que Alfonso VIII encaró la derrota de diversas maneras. En lo político, las disputas internas entre León, Castilla, Navarra y Aragón continuaron, aunque el monarca también comenzó a construir alianzas con los reinos vecinos, principalmente a través de los matrimonios de sus hijas: Berenguela con el rey de León en 1197, Urraca con el rey de Portugal y Blanca con el heredero francés en 1200. La Crónica latina también describe el orgullo con el que Alfonso veía los progresos de su heredero el infante Fernando. Pero, a pesar de todo, "[p]ermanecía fijo en lo profundo de la mente del rey lo que nunca de ella se había borrado: el infortunio que había padecido en la batalla de Alarcos. Muchas veces recordaba en su espíritu aquel día, teniendo deseos de vengarse del rey marroquí, y sobre ello rogaba muchas veces al Señor". ${ }^{17}$ Alfonso VIII trató, además, de compensar a la Orden de Calatrava. En la carta de donación por la que les concedía propiedades en Ronda hacía expreso su lamento por "vuestra pobreza, la pérdida de la casa central de Calatrava y de casi todas vuestras posesiones por el infortunio de Alarcos". ${ }^{18}$

Pero el rey iba a sufrir mayores y más dolorosos reveses antes de la victoria final de Las Navas en 1212. Incapaz de reunir un contingente suficiente para defender la plaza, se vio obligado a rendir el castillo de Salvatierra en septiembre de 1211, aunque sin pérdidas humanas que lamentar. La Crónica latina expresa el dolor por esta nueva derrota en un lenguaje de resonancias bíblicas: "¡Oh, cuánto llanto de hombres y gritos de mujeres gimiendo todas a una y golpeando sus pechos por la pérdida de Salvatierra!". ${ }^{19}$ En octubre de ese mismo año, moría el infante Fernando de unas fiebres, y la crónica recurre de nuevo al modelo bíblico -Jeremías y el Libro de las Lamentaciones- para hacer partícipe al lector del dolor extremo de Alfonso VIII y la reina Leonor tras este trágico suceso. Se cernían así sobre el monarca las mismas desgracias que había sufrido su antepasado Alfonso VI, quien había perdido la batalla de Sagrajas con los almohades en 1086 y a su hijo Sancho en un combate en Uclés en $1108 .{ }^{20}$

La comparación no es baladí, ya que puede servir para sondear la profunda respuesta emocional de ambos monarcas ante esta secuencia de terribles tragedias. La Crónica najerense, escrita probablemente en la abadía de Santa María de Nájera ca. 1173, preserva una semblanza de Alfonso VI en sus horas más bajas que gozará de fortuna posterior, tanto en el De rebus Hispaniae de Rodrigo Jiménez de Rada como en el Chronicon mundi de Lucas de Tuy. ${ }^{21}$ Aunque la derrota de Zallaqa se trata a vuela pluma en la Najerense, se consigna la fecha precisa de la muerte de Sancho en Uclés -el día de la Natividad de san Juan Bautista de 1108- y se aclara que el joven pereció junto a su tutor García Ordóñez y otros seis condes. ${ }^{22}$ Es más, García Ordóñez debió de ser enterrado en Santa María de Nájera. ${ }^{23}$ El siguiente capítulo, con el que se cierra la obra (III.23), dedica sus últimas líneas -después de un pasaje en el que se glorifica a Alfonso VI- a la descripción del gran milagro (magnum fecit miraculum Deus omnipotens) acaecido en San Isidoro de León en la Octava antes de la muerte del monarca. Dicho milagro tuvo lugar en el día de la Natividad de san Juan Bautista, esto es, en el aniversario de la batalla de Uclés y de la muerte de Sancho. Durante cuatro días, el agua brotó de las piedras en torno al altar de San Isidoro, no entre sus junturas sino de los propios sillares (non per iuncturas set per medium petrarum). El agua sólo cesó de manar después de que se celebrase una procesión desde la catedral, seguida de una 
misa, y de que una parte del agua fuese bebida y otra tanta recogida en una vasija de cristal. El cronista aclara en este punto que las piedras lloraron de tal manera que conmovieron hasta las lágrimas a los presentes (Ideoque plorauerunt lapides ut ad fletum homines commouerent) y que aquel hecho fue considerado un signo del dolor y las tribulaciones que caerían sobre España después de la muerte de Alfonso VI (Hoc signum nichil aliud portendebat nisi luctus et tribulationes que Yspanie post eius obitum euenerunt). Pero, dada la elaboración retórica del texto, así como el énfasis puesto en todo lo relativo al aniversario y en el encadenamiento de estas dos desgracias, se hace difícil no ver también en el relato del milagro un lamento por la doble tragedia -la derrota de Zallaqa y, sobre todo, la muerte de Sancho en Uclés- que vino a poner fin al, hasta entonces, brillante reinado de Alfonso VI.

Años más tarde, el castillo de Uclés sería entregado a Alfonso VII como parte del tratado firmado con el rey Lobo de Murcia. A su vez, el monarca castellano lo legaría a su hijo Sancho III, padre del futuro Alfonso VIII. En 1163, cuando este último no había salido aún de la minoría, donaba el castillo a la Orden de San Juan y a la recién creada Orden de Santiago junto a su tío Fernando II de León. ${ }^{24}$ En 1172, por las mismas fechas en que se redactaba la Crónica najerense, Alfonso VIII acrecentaba el número de integrantes de la Orden de Santiago con la incorporación de los freires de Ávila. El acuerdo rezumaba optimismo, ya que se afirmaba que una vez expulsados los moros de España, los freires avanzarían hasta Jerusalén a través de Marruecos. ${ }^{25}$ Dos años después, el castillo de Uclés se había convertido en la sede de la Orden (Caput Ordinis), y se completaba con la construcción de un convento dedicado a la Virgen, protectora de la reconquista. Resulta difícil imaginar cuánto quedaba entonces de aquel triste recuerdo familiar, y tan sólo cabe especular si la decisión de entregar la fortaleza a la Orden de Santiago vino dada por razones puramente estratégicas o si también tuvo como objetivo purgar el pasado y apropiarse simbólicamente de un paisaje marcado por el infortunio.

Como quiera que fuese, todo parece indicar que la derrota de Alarcos había dejado una honda huella emocional en el reino de Castilla que podría justificar la creación de un "memorial de guerra", aunque esta constatación genere nuevos interrogantes. La idea de conmemorar una derrota se antoja extraña en un contexto medieval, puesto que en aquel tiempo el deseo de honrar a los muertos quizás se habría visto mejor satisfecho en la venganza. El reino buscaba cerrar sus heridas pero, ante todo, necesitaba sentir que Dios estaba de su parte. Para conseguir el perdón divino, Castilla debía expiar sus pecados en la persona del monarca.

En los márgenes del agregado literario e historiográfico conformado por la llamada Primera Crónica General o Estoria de España, escrita entre finales del siglo XIII y la primera mitad del siglo XIV, Alfonso VIII aparece retratado como un rey poco ejemplar, que se habría hecho acreedor de la derrota en Alarcos por su romance con la judía Formosa. La primera mención de esta leyenda aparece en una anotación marginal del manuscrito regio de 1289 (Escorial, X.I.4, fol. 291v), aunque la historia debió de ir creciendo de boca en boca. Dicha leyenda afirma, asimismo, que Alfonso mandó erigir el monasterio de Santa María Regalis de Las Huelgas en señal de arrepentimiento. ${ }^{26}$ Dado que no se rastrean referencias contemporáneas a este romance en la lírica trovadoresca ni en la crónica de Jiménez de Rada, figura cercana a la familia real, la historia ha sido rechazada como apócrifa. Sin embargo, aún cuando los hechos relatados por la leyenda puedan ponerse en duda, considero que la noción de penitencia debió de resultar crucial para el desarrollo de la abadía de Las Huelgas. Cabe la posibilidad, incluso, de que la historia de Formosa hubiese servido para ocultar otra realidad más incómoda, la de la debilidad militar de Alfonso VIII al comienzo de su reinado.

En un principio, nada de ello se trasluce en las palabras de Jiménez de Rada, quien sólo vincula la derrota en Alarcos con la fundación de Las Huelgas a través de la yuxtaposición. No obstante, la frase que sigue al relato de la fundación del monasterio afirma que Alfonso mantuvo el recuerdo de la batalla de Alarcos "en lo más profundo de su corazón". ${ }^{27}$ Sin duda, Las Huelgas fue la más importante de las fundaciones religiosas creadas a instancias de Alfonso VIII y la reina Leonor. Erigida en 1187 a las afueras de 
Burgos, la primera reunión anual de su capítulo tuvo lugar en 1189, por lo que es preciso descartar una relación causal entre la fundación de Las Huelgas y la derrota de Alarcos, a la que precedió varios años. ${ }^{28}$ No ocurre lo mismo con el más temprano de sus dos claustros, conocido como Las Claustrillas y datable a finales de la década de $1190 .{ }^{29}$ A este respecto, sugerí en una publicación anterior que este claustro podría haber sido construido bajo el influjo de la pérdida de Jerusalén en 1187, y que su forma habría revelado su función como lugar de enterramiento, no sólo del infante Fernando sino también de otros caídos en Alarcos. ${ }^{30}$ Me gustaría, en cambio, explorar ahora más en detalle la segunda de estas ideas, es decir, la posibilidad de que Las Claustrillas respondiese -al menos en parte- a esa culpa que apesadumbraba a Alfonso VIII.

En buena medida, la documentación proporciona una confirmación de la conexión entre Las Claustrillas y Alarcos, que se encarna en Pedro Rodríguez de Guzmán, uno de los nobles que, de acuerdo con la Crónica latina de los reyes de Castilla, habrían muerto en el campo de batalla. ${ }^{31}$ Él, y posiblemente Ordoño García -otro de los caídos en Alarcos-, aparece entre los firmantes de la carta de fundación de Las Huelgas en 1187.32 En 1194, Pedro Rodríguez de Guzmán y su mujer Mafalda habrían hecho donación de ciertas propiedades al nuevo monasterio pro Dei amore et animarum nostrarum et parentum nostrum redemptione, localizadas éstas en Revilla del Campo, Hontorias y Quintanaseca. Dicha donación debería hacerse efectiva a la muerte del que falleciese primero. ${ }^{33}$ Ya en 1199, cuatro años después de la muerte de Pedro Rodríguez y cuando Las Huelgas pasaron a integrarse definitivamente en la disciplina cisterciense, dos de sus monjas -hijas de este noble castellano- hicieron una nueva donación a la abadía. Siguiendo las disposiciones testamentarias de su padre, ordenaron que su herencia y todos los bienes recibidos de su progenitor así como sus propios cuerpos fuesen entregados a la abadesa de las Huelgas, pro anima de sus padres y de ellas mismas. ${ }^{34}$ Es posible que una de estas hermanas fuese a su vez la viuda de Rodrigo Sánchez, a quien se cree yerno de Pedro Rodríguez. Por su parte, conviene mencionar que sus hermanos Nuño Pérez y
Guillem Pérez habían luchado en Alarcos junto al poderoso Thibaut de Blazón de Poitou quien, de acuerdo con la Crónica latina, era también hijo de Pedro Rodríguez. ${ }^{35}$ Este detalle podría explicar por qué el obispo catalán de Narbona y él fueron los únicos líderes ultrapirenaicos en permanecer junto al rey y unirse a las tropas castellanas en la batalla de Las Navas. ${ }^{36}$

Las hijas de Pedro Rodríguez prosperarían en la abadía: Teresa, presumiblemente la mayor de las dos, aparece en la documentación como cantatrix en 1211, mientras que su hermana María se convertiría en abadesa en 1232. En 1234, la propia María haría una segunda donación a Las Huelgas, por el alma de su hermana y por la suya propia, con el imprimatur del rey Fernando III. ${ }^{37}$ Todo ello invita a pensar que el recuerdo de Alarcos habría sido especialmente intenso en Las Huelgas si, como es de imaginar, fue mantenido vivo durante décadas por las hijas de Pedro Rodríguez, a quien pudo haberse honrado incluso como mártir. Por otro lado, a pesar de que no hay constancia documental de que las donaciones de las dos monjas hubiesen sido destinadas a la construcción del claustro, su ingreso en la abadía coincide con el momento en el que debieron de iniciarse las obras. Estos argumentos no dejan de ser circunstanciales, pero no está de más añadir que la decoración de Las Claustrillas resulta inusualmente ecléctica y disonante, y uno de los motivos que podrían dar razón de esta particularidad es la intervención de varias familias en su decoración.

Parece, pues, que una parte de la abadía de Las Huelgas pudo haber sido construida como acto de expiación por la derrota de Alarcos, y en conexión con la memoria familiar de algunas de las monjas que allí profesaban. Pero, ¿es factible distinguir elementos concretos en la fábrica del monasterio que apoyen esta suposición? La ornamentación escultórica más singular de Las Claustrillas se localiza en el pilar central de la arcada norte, donde aparece figurado el templum domini (Fig. 1). La inclusión de este motivo podría indicar que se pretendió identificar al claustro en su conjunto con el Tabernáculo. ${ }^{38}$ Hace unos años, sugerí que estos pilares intermedios horadados debían interpretarse a la luz de la función funeraria de Las Claustrillas, y sigo pen- 


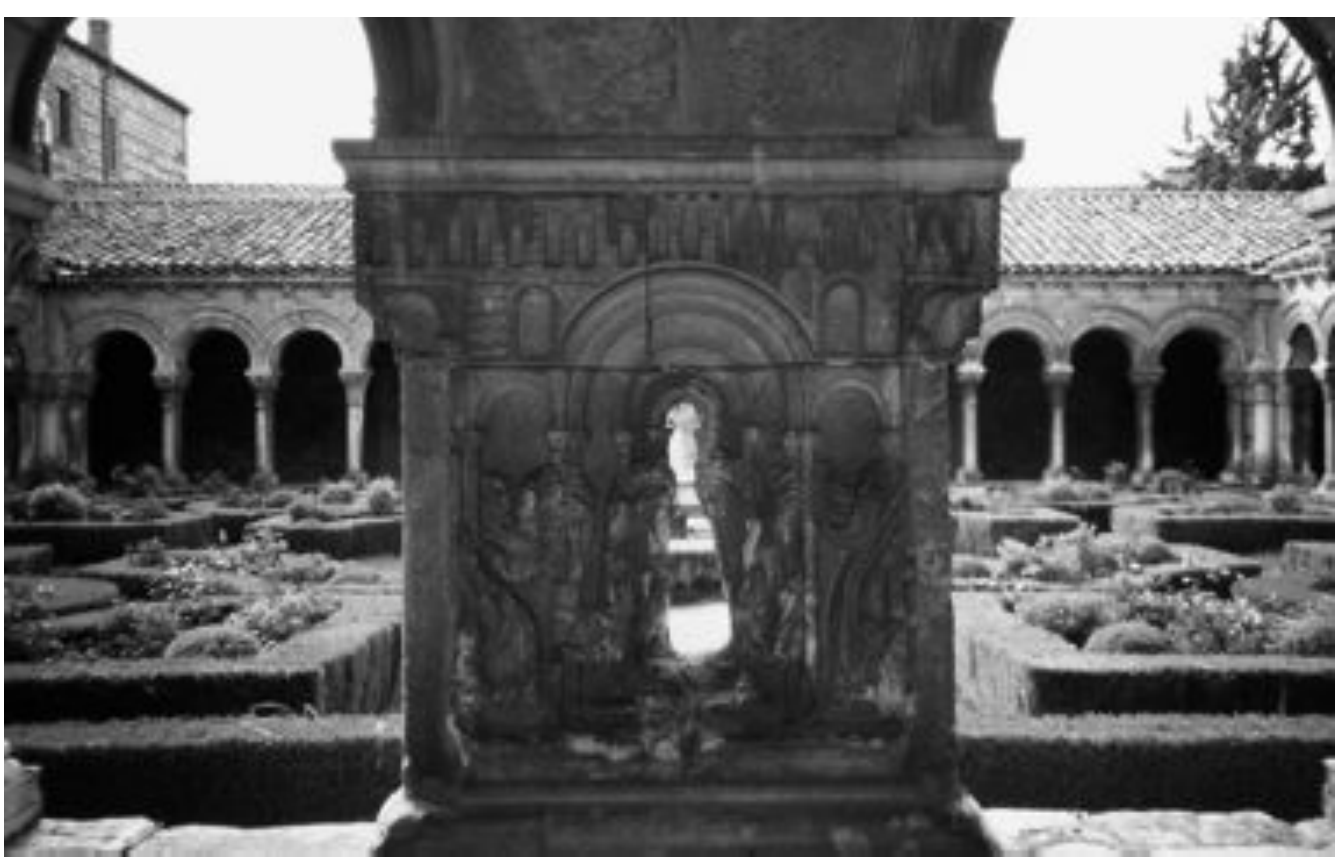

Fig. 1 Detalle del pilar central de la panda norte de Las Claustrillas, Monasterio de Las Huelgas, Burgos (Conway Library, Courtauld Institute of Art).

sando que ése es uno de los niveles de lectura que suscita el análisis del claustro. No obstante, considero ahora que habría que poner mayor énfasis en otra de las hipótesis que se apuntaban en aquel trabajo. Me refiero al hecho de que las representaciones del Templo / Tabernáculo pudiesen haber estado relacionadas con la actividad penitencial, un vínculo apuntado por Mary Carruthers. ${ }^{39}$ Esta autora ha analizado el modo en el que la medición del Tabernáculo se convirtió en una actividad penitencial durante la Edad Media, sugerida por el texto que recoge el Libro de Ezequiel en su capítulo 43:

Mas tú, ioh hijo del hombre!, muestra a los de la casa de Israel el templo, y confúndanse de sus maldades; y midan la fábrica. $Y$ avergüéncense de toda su conducta: muéstrales la figura de la casa, las salidas y entradas del edificio, y todo su diseño, y todas sus ceremonias, y el orden que debe observarse en ella, y todas sus leyes; y lo escribirás todo a la vista de ellos, para que observen todo el diseño que se da de ella, y sus ceremonias, y las pongan en práctica (Ez. 43: 10-11).
Carruthers sostiene que la "medida de la fábrica" de la Ciudad Celestial se convirtió en un tropo de la "meditación penitencial" y un tópico de la retórica monástica. Así, el texto bíbli$\mathrm{co}$, que funcionaba como "ritual de penitencia para Israel y de reconciliación con Dios" con el fin de alcanzar la "alianza de salvación", habría podido servir de ejemplo para una penitencia a nivel nacional, respondiendo a la perfección a esa necesidad que tenía Castilla de reconciliarse con el Todopoderoso. La tradición exegética surgida a partir del pasaje de Ezequiel, consideraba esta penitencia como un proceso activo ya que, como refería Rabano Mauro, mientras "medimos la fábrica" debemos avergonzarnos por nuestros pecados hasta que el rubor coloree nuestras mejillas. Esta interpretación más personal se habría ajustado a la clase de expiación que reclamaban los errores de Alfonso VIII en la campaña de Alarcos. Sin duda, este "medir la fábrica" habría sido la actividad penitencial más adecuada para purgar un pecado que era al mismo tiempo personal y social.

Pero para Carruthers se trataría también de "un acto de contrición, expresado por medio de 
un esquema mnemónico arquitectónico". ${ }^{40}$ Esta tarea (con)memorativa podía abordarse mediante una imagen bidimensional como el plano del Tabernáculo en el Codex Amiatinus (Florencia, Biblioteca Medicea Laurenziana, ms. Amiatinus I, ff IIv-III), donde el ojo de la mente puede errar a su antojo entre cortinajes y columnas. ${ }^{41}$ Asimismo, podía comportar además todo un ceremonial litúrgico, el sacramento de la penitencia y actividades públicas como procesiones. El ductus de la labor memorativa podría incluso llevar al penitente a deambular y caminar a través de un espacio tridimensional como un claustro monástico y, en especial, por uno como Las Claustrillas, con sus pilares horadados, columnas y cortinas labradas. ${ }^{42}$ Como quiera que fuese, este proceso de medición bíblico tenía la facultad de "reconciliar a toda la comunidad con Dios". ${ }^{43}$ Después de la derrota de Alarcos, Alfonso VIII anhelaba esa reconciliación, no sólo a nivel individual sino también en lo que atañía al reino de Castilla como colectividad. Sin embargo, a pesar de lo apropiada que hubiese podido resultar esta tarea conmemorativa en el contexto inmediatamente posterior a la derrota, conviene rastrear alguna evidencia que permita certificar que tal actividad penitencial tuvo lugar en Las Huelgas. En este sentido, la Primera Crónica General de España ofrecería un primer testimonio a favor de esta conjetura, al afirmar que las santas vírgenes consagradas que estaban "ençerradas alli en la claustra et en la eglesia", debían "alabar et cantar alli a Dios de dia et de noche" para pedir en nombre de los cristianos que los "ampa[ra]re yl enderesç[ar]e al su servitio y que a su diestra parte". ${ }^{44} \mathrm{El}$ uso del verbo enderesçar y la mención de la diestra divina realzan las connotaciones "correctivas" del pasaje, de lo que se deduce que la liturgia de las monjas tenía la capacidad de interceder por aquello de lo que los ejércitos castellanos estaban tan necesitados, el favor de Dios.

Con todo, es la ornamentación escultórica de Las Claustrillas donde se halla confirmación adicional de esta sospecha, en tanto que expresión concreta de la actividad penitencial que tenía lugar entre sus muros. Como se indicó antes, el claustro pudo haber sido considerado una imagen del Tabernáculo, y tal vez la existencia en cada panda de seis arquerías a izquierda y derecha de los pilares intermedios haya venido dada por algún tipo de simbolismo numérico, habida cuenta que el número seis juega un papel preponderante en las medidas del Templo en el Libro de Ezequiel. Sin embargo, habría sido en el pilar intermedio del lado norte donde la alusión al Templo se habría hecho explícita. En su cara sur, cuatro órdenes de columnas enmarcan el vano del pilar, rodeadas a su vez por un ondeante cortinaje. Corona el conjunto una suerte de dintel rematado en sus extremos por sendas estructuras inusuales que deliberadamente evocan una arcada finestrada. En la cara norte, la que da al patio, no es posible determinar si las columnas y los cortinajes no llegaron a labrarse o fueron destruidos pero se despliega la ornamentación micro-arquitectónica de un modo similar (Fig. 2).

Esta identificación con el Tabernáculo se ve reforzada al comparar la decoración del pilar con una de las ilustraciones del Beato de Cardeña (Madrid, Museo Arqueológico Nacional, ms. 2,

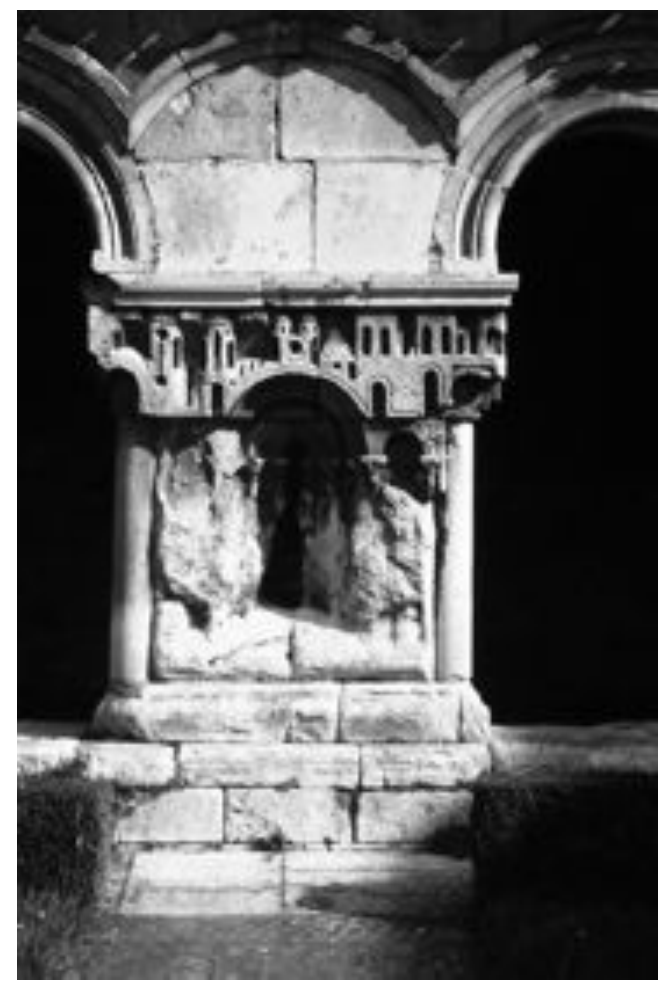

Fig. 2 Detalle de Las Claustrillas, Monasterio de Las Huelgas, Burgos (Conway Library, Courtauld Institute of Art). 


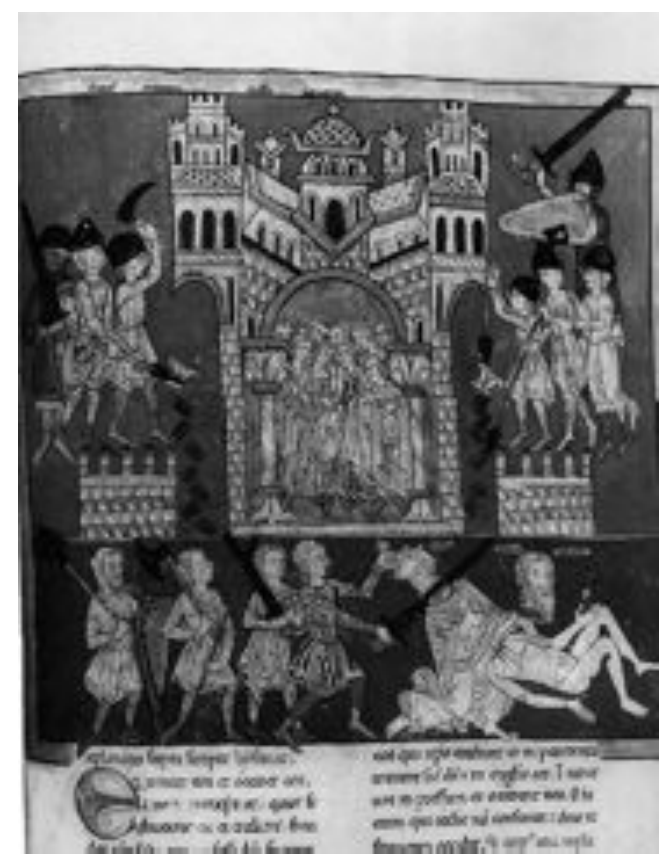

Fig. 3 llustración del Beato de Cardeña. Madrid, Museo Arqueológico Nacional, MS 2, fol.106 (Arxiu Mas).

fol. 106; Fig. 3), al que habitualmente se atribuye una cronología ca. 1180 en función de criterios estilísticos. No obstante, las estrechas semejanzas que se detectan entre estas dos obras aconsejan retrasar su confección a una fecha posterior a la caída de Jerusalén (1187) o, incluso, en la década de $1190 .{ }^{45}$ La rúbrica que acompaña a la miniatura identifica al Anticristo arrasando la ciudad de Jerusalén así como el asesinato de los dos testigos de la desgracia, Enoc y Elías. Estas escenas se disponen en dos registros, ocupando la primera de ellas el nivel superior. En él, los soldados cargan contra Jerusalén y, de hecho, empiezan ya a demoler los muros de la ciudad, que es figurada como un gran arco -apoyado sobre dos columnas- bajo el que se cobija la población. Más arriba, las micro-arquitecturas se prolongan hasta rematar en dos torres que recuerdan a los peculiares remates de los pilares de Las Huelgas, un detalle tan inusual que sugiere que ambas imágenes fueron concebidas en el mismo ámbito, por lo que no es de extrañar que se haya propuesto un origen burgalés para el manuscrito -más concretamente el monasterio de San Pedro de Cardeña- y hasta la posibilidad de que, quizás, se debiese al patronazgo de Alfonso VIII y Leonor. ${ }^{46}$ Por si todo ello fuera poco, el Beato Rylands (Manchester, John Rylands University Library, MS lat. 8, fol. 134v) se sirve de un encuadramiento arquitectónico similar para ilustrar el pasaje del Apocalipsis donde Juan mide el Templo del Señor con una vara, lo que ha de considerarse otro argumento de peso a favor de la hipótesis que aquí se propone, es decir, que Las Claustrillas fueron concebidas en el rico contexto litúrgico y político de la pérdida de Jerusalén y la derrota de Alarcos. ${ }^{47}$ Lejos de ser un mero reflejo de estos dos eventos, el claustro habría jugado un papel activo en el recuerdo y conmemoración litúrgica de ambos hechos luctuosos, al ofrecer a las monjas un lugar de constante meditación, plegaria y rezo, Las Huelgas se habría convertido en la fortaleza espiritual desde la que se guiaría a Castilla hacia la victoria en Las Navas. Como cantorix, Teresa Pérez podría haber supervisado gran parte de esta intensiva labor litúrgica y musical. Además de generar un entorno favorable para futuras campañas, la actividad penitencial habría circunscrito las memorias de Alarcos al espacio arquitectónico de Las Claustrillas, liberando a Alfonso VIII y a sus colaboradores del peso de la derrota para poder concentrarse en el análisis de los errores cometidos entonces y en la cuidadosa preparación de la ofensiva, una vez finalizado el tratado de paz con los almohades. ${ }^{48}$

Ciertamente, como señalan aquellos que trabajan sobre monumentos conmemorativos de la Edad Moderna y Contemporánea, la arquitectura puede funcionar más como instrumento para el olvido que para la memoria, puesto que acaba por suplantar al propio recuerdo. ${ }^{49}$ Las Claustrillas ofrecen un elocuente ejemplo de que este fenómeno no era ajeno tampoco a la Edad Media. Pero, ¿es esta evidencia suficiente para considerar este conjunto como un memorial de guerra en algún sentido? En mi opinión, la respuesta a esta pregunta ha de ser afirmativa. El claustro burgalés constituía una rareza, en tanto que conmemoración de una derrota, y tenía un carácter dinámico, al estar concebido para su propia superación. Sin embargo, Las Claustrillas diferían en un aspecto de los monumentos conmemorativos de los siglos XIX y XX, ya que no se trataba de un espacio público, sino privado. La interacción 
y la performance quedaban reservadas fundamentalmente a las monjas y los capellanes de la abadía, así como, quizás, a Alfonso VIII, la reina Leonor y otros miembros de la familia real. Sus muros pudieron acoger también ceremonias en recuerdo de uno o más mártires de Alarcos -Pedro Rodríguez y su yerno Rodrigo Sánchezpero, de ser así, se trató de eventos privados. Por el contrario, ¿hubo algún tipo de memorial para la multitud de caídos en la batalla, al que pudiera tener acceso cualquiera con independencia de su condición? Hay varias respuestas posibles para esta pregunta, y casi todas ellas nos conducen a las Órdenes Militares.

Sin duda, la contribución de los soldados individuales no dejó de ser tenida en cuenta, y el propio Jiménez de Rada así lo reconoce después de la victoria de Las Navas, al poner en su boca una advertencia destinada al monarca: "Acordaos de vuestros hombres, sin cuya ayuda no habríais alcanzado tan gran victoria". ${ }^{50}$ Son estos soldados los que aparecerían labrados en un capitel de San Pedro de Gumiel en Izán (Burgos). San Pedro de Gumiel era el monasterio donde Diego Velázquez -uno de los primeros miembros de la Orden de Calatrava- se refugió tras la derrota de Alarcos, y allí sería enterrado en 1196. Jiménez de Rada lo recuerda en su crónica con respeto y puede que con afecto. ${ }^{51}$ Su cuerpo sería objeto de culto varios años después. ${ }^{52}$ Pero San Pedro de Gumiel se convertiría también en un lugar de conmemoración para la Orden de Calatrava en los años que siguieron a la batalla de Alarcos. Por ello, es preciso llamar la atención sobre los nítidos vínculos que cabe establecer entre los motivos vegetales labrados en uno de los pilares de esquina de las Claustrillas y la escultura del cenobio de Izán. ${ }^{53}$ No obstante, en San Pedro de Gumiel se halla otra escultura que nada tiene que ver con la de Las Huelgas, ni con ninguna obra que haya sido etiquetada como "arte de Cruzada". ${ }^{54}$ En especial, destacan las ocho cabezas labradas con un relieve de bulto en el centro de uno de los capiteles (Fig. 4). Algunas muestran sombreros con visera mientras que otras se cubren con yelmos más elaborados, que incluyen una protección por debajo de la barbilla. Esta composición difiere de las representaciones de caballeros victoriosos, mucho más difundidas. ${ }^{55}$ En su sencillez, que parece

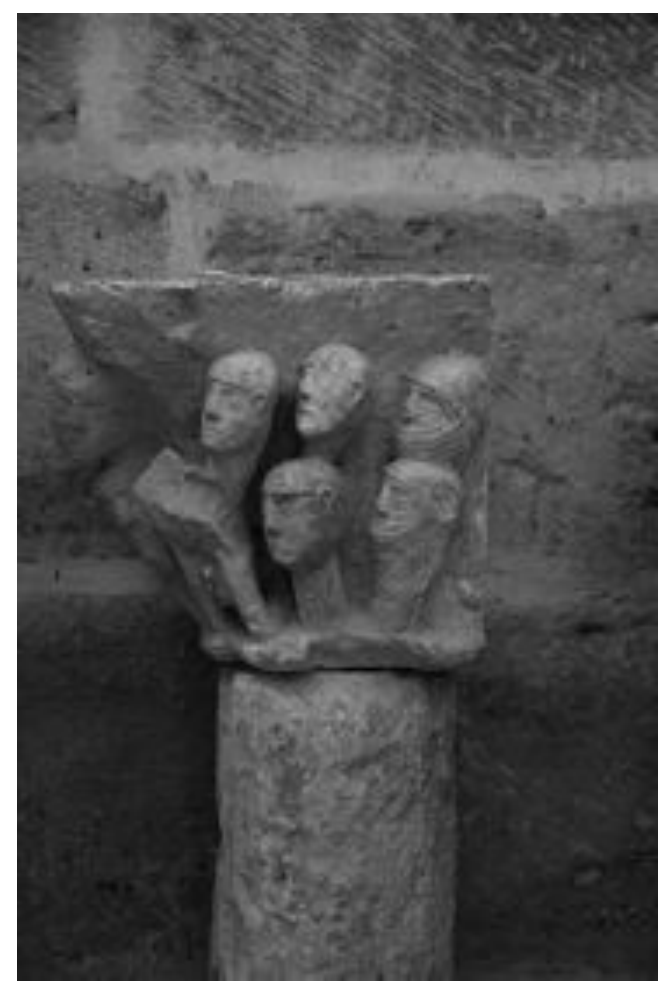

Fig. 4 Capitel de San Pedro de Gumiel de Izán, Burgos (John Batten Photography).

más propia del arte del siglo XX, el conjunto sugiere la idea de una cierta hermandad entre los retratados, probablemente fallecidos. No sería éste el único lugar de memoria para la Orden, puesto que habría que añadir también los de Zorita de los Canes en Guadalajara y Alcañiz. El caso de Montsalud resulta más problemático; allí había sido enterrado Nuño Pérez de Quiñones, el maestre de Calatrava fallecido en Alarcos. ${ }^{56}$ Del mismo modo que los cuerpos de los freires descansaban desmembrados, la memoria de los calatravos caídos se dispersaba.

Durante años, el campo de Alarcos debió de ser el único sepulcro que acogiese a los miles de soldados anónimos que allí habían encontrado la muerte, a pesar de que textos posteriores señalen que se había erigido una capilla para honrarlos, Nuestra Señora de los Mártires. ${ }^{57}$ Hoy en día, su visión trae a la memoria las palabras de Victor Hugo a propósito del campo de Waterloo, "[n]o hay tumba francesa. Para Francia es sepul- 


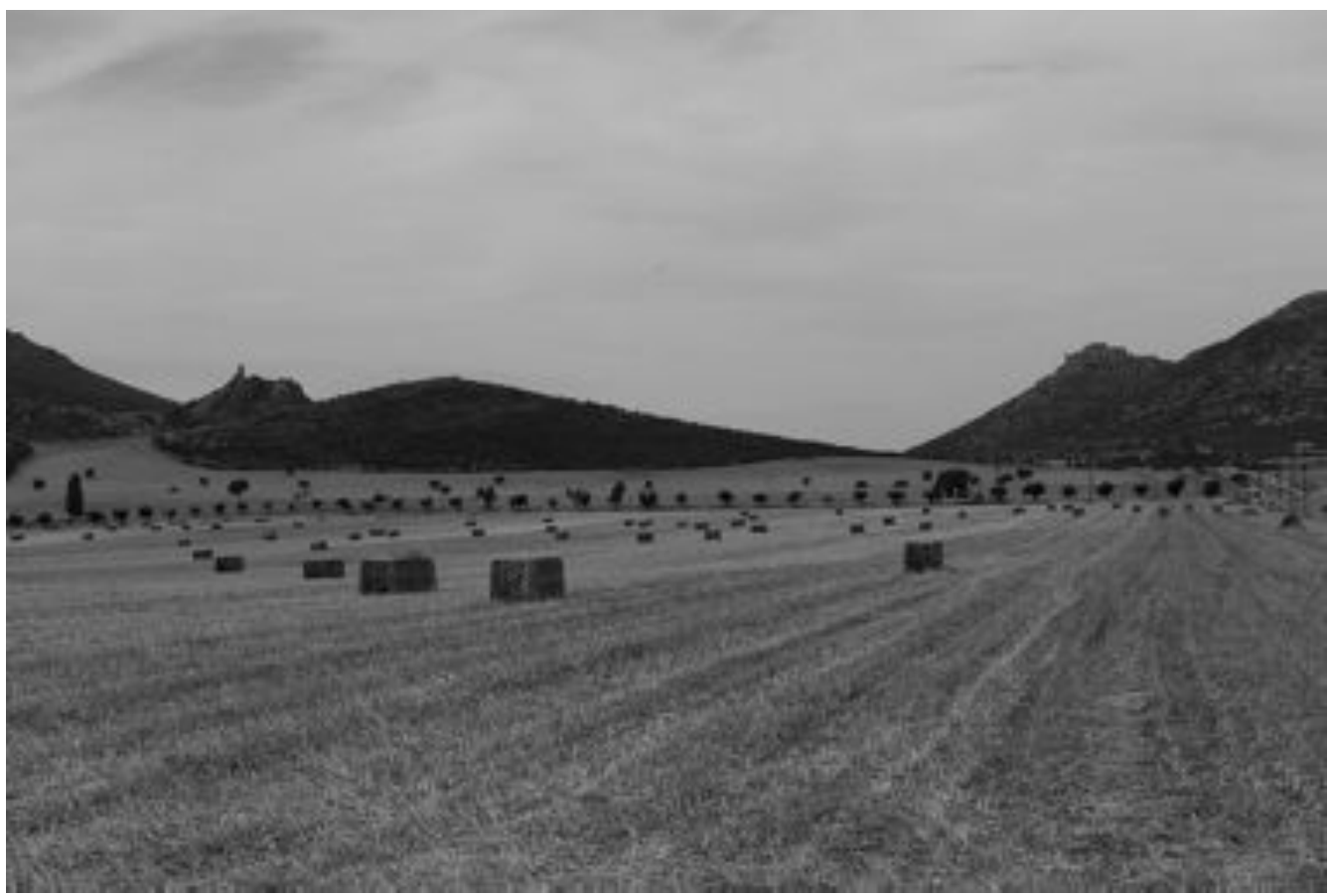

Fig. 5 Vista del paso protegido por la fortaleza de Salvatierra, Ciudad Real (John Batten Photography).

cro toda esta llanura". Pero en el siglo XIII la memoria de Alarcos habría de quedar unida para siempre a la del pequeño grupo caído en Las Navas, al concentrarse los restos de unos y otros en el paso protegido por la fortaleza de Salvatierra, la vía hacia Andalucía (Fig. 5). El paisaje entero debía de estar impregnado todavía de la memoria de las campañas y de los muertos y, no por casualidad, sería este lugar el que se perpetuase como "memorial de guerra" público. El paso es todavía hoy un enclave extraordinario, protegido por las ruinas del castillo de Salvatierra al este y la más sólida fortaleza de Calatrava la Nueva al oeste. Salvatierra había sido la ciudadela desde la que los caballeros calatravos habían salido a la batalla para luchar y morir en Alarcos. Su pérdida había puesto el doloroso broche simbólico al annus horribilis de 1211, dejando de ser el "castillo de salvación" para convertirse, en palabras del historiador musulmán del siglo XIV Ibn Idari, en "el lugar de expiación de los pecados" para los calatravos. Tal vez por ello, a pesar de ser recuperado dos décadas después, no volvería a servir de emblema a la Orden como

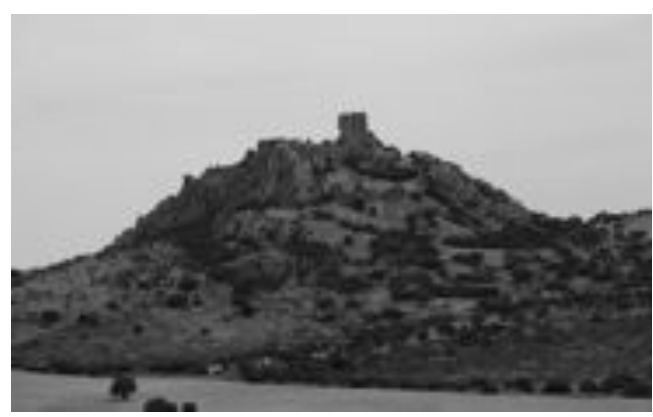

Fig. 6 Restos del castillo de Salvatierra, Ciudad Real (John Batten Photography).

depositario de su memoria e identidad (Fig. 6). Desde 1217 tal función vendría a ser desempeñada por el nuevo castillo, Calatrava la Nueva, levantado en el lado opuesto del desfiladero. ${ }^{58}$ Allí fueron trasladados los restos de los muertos en Alarcos y Las Navas. De este modo, los caídos en uno y otro enfrentamiento fueron honrados como mártires en un monumento conjunto, un cementerio de diseño claustral construido sobre una elevada plataforma, con el que se habría sacralizado el nuevo emplazamiento. También 
se erigió una capilla en el lugar, dedicada a la Virgen -Nuestra Señora de los Mártires- y fueron muchos los maestres de Calatrava que decidieron enterrarse ad sanctos (Fig. 7). ${ }^{59}$ La Virgen habría de presidir así el recuerdo y la intercesión por los miembros de la Orden, ya fuesen mártires de Alarcos o de Las Navas. En este sentido, considero que es legítimo ver en la nueva capilla

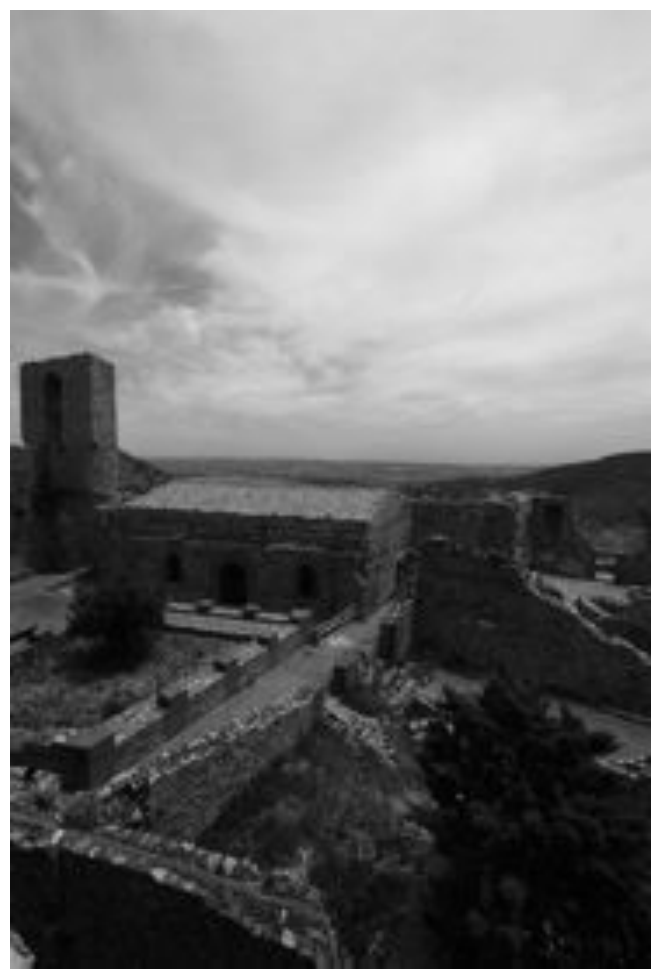

Fig. 7 Restos del castillo de Calatrava la Nueva, Ciudad Real (John Batten Photography). un memorial de guerra para la Orden en tanto que confraternidad, que habría seguido funcionando como tal durante siglos y encarnando estas memorias que constituían una parte crucial de la identidad calatrava.

En Las Huelgas de Burgos, el doloroso recuerdo de Alarcos había sido resuelto y asimilado, en apariencia como consecuencia de la actividad penitencial desarrollada allí durante décadas. Bajo la protección de la Virgen, Santa María Regalis de Las Huelgas terminó por metamorfosearse en lugar de la memoria de la victoria en Las Navas de Tolosa. Como Rocío Sánchez Ameijeiras ha demostrado con argumentos que no dejan lugar a duda, la gesta fue celebrada en la abadía con la Fiesta de la Exaltación de la Santa Cruz. La liturgia incorporaba incluso fragmentos del De rebus Hispaniae de Jiménez de Rada. ${ }^{60}$ Alfonso VIII se había convertido en un rey victorioso, festejado por toda la Cristiandad, a pesar de que su muerte en 1214 le privó bien pronto de disfrutar de este merecido homenaje. Para entonces, en Las Huelgas se iniciaba un proceso de reforma más acorde con las coordenadas estéticas del gótico, y su claustro tardorrománico -Las Claustrillas- habría de quedar arrinconado ante el nuevo e imponente espacio claustral. Pero, en los confines del antiguo recinto que tantos recuerdos atesoraba, la pequeña capilla de la Asunción, donde reposaron en un primer momento los cuerpos de Alfonso VIII y Leonor, sería cubierta también con una nueva cúpula y una delicada ornamentación en estuco digna de los derrotados soberanos almohades. ${ }^{61}$ La memoria volvía a revestirse con los atributos tradicionales de la celebración de la victoria. 


\section{NOTAS}

${ }^{1}$ Deseo expresar mi agradecimiento a Tom Nickson por haberme dado la oportunidad de presentar este trabajo en el congreso "Constructing Memory in Medieval Spain", celebrado en la Universidad de York en 2011.

2 CARRUTHERS, M., The Craft of Thought. Meditation, Rhetoric, and the Making of Images, 400-1200, Cambridge, 1998, 35-40. Véase también ROWLANDS, M., "Remembering to Forget: Sublimation as Sacrifice in War Memorials", en The Art of Forgetting, FORTY, A. y KÜCHLER, S., Oxford, 1999, 129-146.

3 CLARKE, J., "The Sacred Names of the Nation's Dead: War and Remembrance in Revolutionary France", en Memory, Mourning, Landscape, ANDERSON, E., MADDRELL, A., MCLOUGHLIN, K., VINCENT, A., (eds.), Amsterdam and New York, 2010, 21-42.

${ }^{4}$ Para una aproximación al estudio de los memoriales de guerra, véase BORG, A., War memorials: from antiquity to the present, London, 1991 e idem, "Some Medieval War Memorials", en Medieval Architecture and its intellectual context, studies in honour of Peter Kidson, FERNIE, E. y CROSSLEY, P. (eds.), London, 1990, 1-7.

${ }^{5}$ HALLAM, E. M., "Monasteries as 'War Memorials': Battle Abbey and La Victoire," en The Church and War, Studies in Church History, 20, 1983, 47-57; esp. 53; FERNIE, E., The Architecture of Norman England, Oxford, 2000, 102-03.

6 JANAUSCHEK, P.L., Originum Cisterciensium, vol.1, Vindobonae, 1877, 110.

7 SMITH, C., Christians and Moors in Spain, vol. 3. Arabic Sources (7111501), Warminster, 1992, 134-137.

${ }^{8}$ Chronica latina regum CasteIlae, CHARLO BREA, L., ESTÉVEZ SOLA, J. A., y CARANDE HERRERO, R. (eds.) Chronica hispana saeculi XIII. Corpus christianorum. Continuatio medievalis 73, Turnhout, 1997, 44-47, caps. 12-13. La traducción castellana puede consultarse en Crónica latina de los reyes de Castilla, CHARLO BREA, L. (ed.), Madrid, 1999, 37-9, caps. 12-13.
9 VANN, T. M., "Battle of Alarcos", en Medieval Iberia. An encyclopaedia, GERLI, E. M. (ed.), New York, 2003, 42-3. O'CALLAGHAN, J. F., Reconquest and Crusade in Medieval Spain, Philadelphia, 2003, 61.

${ }^{10}$ Crónica latina, 39.

11 LOMAX, D., La Orden de Santiago (1170-1275), Madrid, 1965, 90; O'CALLAGHAN, J. F., "The Order of Calatrava: Years of Crisis and Survival, 1158-1212," en The Meeting of Two Worlds: Cultural Exchange Between East and West During the Period of the Crusades, Kalamazoo, 1986, 419-430.

12 ANIZ IRIATE, C. and DÍAZ MARTíN, L. V., Santo Domingo de Caleruega en su contexto socio-político, 11701221. Jornadas de Estudios Medievales, Salamanca, 1994, 199. La muerte de Pedro Rodríguez de Guzmán el 18 de julio de 1195 también quedó registrada en el obituario de la catedral de Burgos. Véase SERRANO, L., El obispado de Burgos y Castilla primitiva desde el siglo V al XIII, Madrid, 1935, 385.

13 O'CALLAGHAN, Reconquest, 61-2.

${ }^{14}$ MILÁ y FONTANALS, M., De los trovadores en España: Estudio de lengua y poesía provenzal, Barcelona, 1861, 114-116.

15 MATTHEW PARIS, Chronica majora, Rerum britannicarum medii evi scriptores 57, vol. 2, 1067-1216, LUARD, H. R., (ed.), London, 1872-83, 410: De horribili adventu paganorum in Hispaniam. ... tota Christianitas contremuit conturbata.

${ }^{16}$ RODRIGO JÍMENEZ DE RADA, Historia de rebus Hispanie sive Historia Gothica, FERNÁNDEZ VALVERDE, J. (ed.), Corpus Christianorum. Continuatio Mediaevalis 72, Turnhout, 1987, 8.10: "Estote memor gracie Dei, que omnes deffectus in uobis suppleuit et oprobrium aliquandiu toleratum hodie releuauit".

17 Crónica latina, 36-37.

18 GONZÁlEZ GONZÁLEZ, J., El reino de Castilla en la época de Alfonso VIII, Madrid, 1960, 2, 164-66, doc. 658: condolens paupertate eo quod domum uestrum maiorem de Calatraua uestrasque omnes fere res ex infortunio de Alarcos. Véase también RADES Y ANDRADA, F., Crónica de las tres Órde- nes de Santiago, Calatrava y Alcántara (facsímil con un estudio sobre "La obra histórica de Rades y Andrada" por LOMAX, D.W.), Biblioteca de Historia Hispánica, Órdenes Militares, Serie Maior 2, Barcelona, 1980.

19 Crónica latina, 45-6.

20 SMITH, C., Christians and Moors in Spain, vol.3. Arabic Sources (7111501), Warminster, 1992, 92-99.

${ }^{21}$ ESTÉVEZ SOLA, J., Chronica Hispana saeculi XII, Pars II: Chronica Naierensis, Corpus Christianorum, Continuatio Medievalis, LXXI, Turnhout, 1995.

22 Ibid., 3. 21-22: ubi etiam occisus est comes Garsias de Grannione cognomento Crispus et sex alii comites cum eo.

${ }^{23}$ REGLERO DE LA FUENTE, C. M., "La Crónica najerense, Santa María de Nájera y Cluny", e-Spania [Online], 7 junio 2009, Online desde 1 julio de 2009, consultada el 26 de agosto de 2012. URL: http://e-spania.revues.org/18162; DOI: 10.4000/e-spania.18162, § 63.

24 RIVERA GARRETAS, M., "La orden de Santiago en Castilla la Nueva en los siglos XII y XIII", en Las Órdenes Militares en el Mediterráneo occidental: Siglos XIII-XVIII: coloquio celebrado los días 4, 5 y 6 de mayo de 1983, Madrid, 1989, 23-40.

${ }^{25}$ O'CALLAGHAN, Reconquest, 5.

${ }^{26}$ DYER, N.J., "Alfonsine Historiography: The Literary Narrative", en Emperor of Culture. Alfonso X the Learned of Castile and His Thirteenth-Century Renaissance, BURNS, R.I., (ed.), Philadelphia, 1990, 141-158; DEYERMOND, A.D., A Literary History of Spain. The Middle Ages, London and New York, 1971, 88-89; MENÉNDEZ PIDAL, R., Primera Crónica de España, vol. 2, Madrid, 1977, 685 (cap. 1006): El muy noble rey da Alffonsso tiene condesada en el muy alto su coraçon la batalla de Alarcos, et en lo que guisarlo pudiese, querisse uengar, como prinçep de grand poder et de muy grand coraçon, en qualquiera manera et en qualquiera logar que lo el guisar pudiesse, de lo que fuera maltrecho en a quella batalla de Alarcos (...) començo a laurer et a fazar çerca Burgos un monesterio de duennas de la orden de Çesteles. 
27 De rebus Hispaniae, 7.33.

28 WALKER, R., "Leonor of England, Plantagenet queen of King Alfonso VIII of Castile, and her foundation of the Cistercian abbey of Las Huelgas. In imitation of Fontevraud?", Journal of Medieval History, 31 (2005) 346-368.

${ }^{29}$ WALKER, R., "The Poetics of Defeat: Cistercians and Frontier Gothic at the Abbey of Las Huelgas", en HOURIHANE, C., (ed.), Spanish Medieval Art: Recent Studies, Tempe, 2007, 187-213, esp. 189-195.

30 Ibid., 212-213.

31 Crónica latina, 39 (cap. 13): "en la primera línea de de los cristianos caen importantes hombres: Ordoño García de Roda y sus hermanos, Pedro Rodríguez de Guzmán y Rodrigo Sánchez, su yerno, y bastantes otros muchos."

32 LIZOAIN GARRIDO, J. M., Documentación del monasterio de las Huelgas de Burgos (1116-1230), vol.1, Burgos, 1985-90, no.11, 19-23.

33 LIZOAIN GARRIDO, Documentación, no. 36, 66-67. También la propiedad denominada Riofocin, que había sido entregada a sus hijas Teresa y María, habría de volver a manos de sus hermanos o descendientes a la muerte de éstas.

${ }^{34}$ LIZOAIN GARRIDO, Documentación, no. 50, 87-89: pro divisione quod fecit pater noster, dompnus Potro Roderici de Guzman, pro anima eius et pro animabus nostris donamus [... a la abadesa Maria Gutierrez y sus sucesoras ...] totam illam nostram hereditatem, quantum habemus et habere in Ribilla del Campo et in Fontorias, cum quantum nobis ibi pertinet ex parte patre nostro Petro Roderici de Guzman ... cum corporibus nostris iure heditario in perpetuum. El documento es confirmado por el infans Fredinandus, por el mayordomo de la reina, así como por su hermano Nuño Pérez. Véase también ANIZ IRIATE, Santo Domingo, 199-201.

35 GÓMEZ, M.D., The Battle of Las Navas de Tolosa: the Culture and Practice of Crusading in Medieval Iberia, Unpubl. PhD. Dissertation, University of Tennesse-Knoxville, 2011, 124-126. Jiménez de Rada (De rebus Hispaniae,
8.6.50-2), confirma que fue un "hombre noble y valeroso, hispano de nacimiento y castellano de familia" (homo nobilis et strenuus et natione Hispanus et genere Castellanus). Resulta un tanto extraño que Thibaut pudiese haber sido al tiempo hijo del castellano Pedro Rodríguez y señor de Blazón. Una posibilidad es que la conexión se verificase a través de su madre Mafalda. Pedro Rodríguez y Mafalda se habían casado en 1174, cuando Alfonso VIII ofreció a ésta un regalo de boda (in casamento). Véase GONZÁLEZ, El reino, 2, 338-339, doc. 205.

${ }^{36}$ Crónica latina, 51 (cap. 22): "se quedaron unos pocos con Teobaldo de Blazón, hijo de Pedro Rodríguez de Guzmán, y con el arzobispo narbonense, que era oriundo de Cataluña".

37 LIZOAIN GARRIDO, Documentación: no.106, 170-171, donde Teresa Peidrez, cantatrix confirma después de la prioresa; ibid. Documentación, vol. 3, 1263-1283, no. 276, 40-41: donna Maria Perez de Guzman, por la gracia de Dios abbadessa del monesterio de Sancta Maria la Real de Burgos, do por mi alma e de donna Teresa, mi hermana.

$$
38 \text { WALKER, "The Poetics", 210- }
$$
211.

39 Ibid., 213. CARRUTHERS, 231237.

40 CARRUTHERS, 234-237 and pl. 24.

${ }^{41}$ REVEL-NEHER, E., "Du Codex Amiatinus et ses rapports avec les plans du Tabernacle dans l'art juif et dans l'art byzantine", The Journal of Jewish Art, 9 (1982) 6-17

${ }^{42}$ CARRUTHERS, The Craft, 27276.

43 Ibid., 233.

44 MENÉNDEZ PIDAL, Primera Crónica General, 685 (cap. 1006): porque las sanctas virgenes conssagradas, que alabassen et cantassen alli a Dios de dia et de noche non sintiessen ninguna mingua de todas casas ouiessen mester: mas que, ençerradas alli en la claustra et en la eglesia et en las otras moradas (...) en cantar a Dios et alabarle et pedirle merced por su pueblo cristiano que le ampare $y$ l enderesce al su servitio y que a su diestra parte.
${ }^{45}$ WILLIAMS, J., The Illustrated Beatus. A Corpus of the Illustrations of the Commentary on the Apocalypse, vol. V, The Twelfth and Thirteenth Centuries, London, 2003, 24-30 and fig. 168.

46 Ibid., 24-27. WALKER, R., "Leonor of England and Eleanor of Castile: Anglo-lberian marriage and cultural exchange in the twelfth and thirteenth centuries", en BULLÓN-FERNÁNDEZ, M. (ed.), England and Iberia in the Middle Ages, 12th-15th century. Cultural, literary, and political exchanges, New York, 2007, 72-73.

47 WILLIAMS, The Illustrated Beatus, 19-23, and fig. 68.

${ }^{48}$ ALVIRA CABRER, M., "De Alarcos a las Navas de Tolosa: Idea y realidad de los orígenes de la batalla de 1212", en Actas del Congreso Internacional Conmemorativo del VII Centenario de la Batalla de Alarcos, IZQUIERDO BENITO, R. y RUÍZ GÓMEZ, F. (eds.), Ciudad Real, 1996, 249-264, esp. 254.

${ }^{49}$ FORTY, A. and KÜCHLER, S., The Art of Forgetting, Oxford, 1999.

${ }^{50}$ De rebus Hispaniae (8.10.49): Toletanus pontifex hec uerba dixit nobili regi: "... Estote etiam memor uestrorum militum, quorum auxilio ad tantam gloriam peruenistis".

51 Ibid., 7.14: Didacus Velasqui postea diu vixit, quem etiam memini me vidisse, et obit in Monasterio Sancti Petri de Gumiello, et ibi sepultus est, requiescat in pace.

52 MATEO, C., "Fray Diego de Velásquez (1196-1996)", Cistercium, Historia, Arte, Espiritualidad. Revista monastica, 205 (1996) 323.

53 WALKER, "The Poetics", 198204; por lo que respecta a Gumiel, véase PÉREZ CARMONA, J., Arquitectura y escultura románicas en la provincia de Burgos, Burgos, 1959, 265-66, fig. 288 у 289.

54 Véase, por ejemplo, FOLDA, J., Crusader Art: the art of the Crusaders in the Holy Land, 1099-1291, Aldershot, 2008; GÓMEZ, M. D., "The Crusades and church art in the era of Las Navas de Tolosa", Anuario de historia de la iglesia, 20 (2011) 237-60.

${ }^{55}$ RUIZ MALDONADO, M., "El «caballero victorioso» en la escultura 
románica española. Algunas consideraciones y nuevos ejemplos", Boletín Seminario de Estudios de Arte y Arqueología, 1979, 271-286.

${ }^{56}$ LAYNA SERRANO, F., La arquitectura románica en la provincia de Guadalajara, Guadalajara, 2001, 180183, 185-189.

57 ZAPATA ALARCÓN, J., "El culto a los mártires: Visión y símbolo del medievo a la contrarreforma. La construc- ción del relicario del Sacro Convento de Calatrava la Nueva", en Actas del Congreso Internacional Conmemorativo del VII Centenario de la Batalla de Alarcos, IZQUIERDO BENITO, R. y RUÍZ GÓMEZ, F. (eds.), Ciudad Real, 1996, 615-23.

58 Ibid. 616.

59 Ibid., 616.

60 SÁNCHEZ AMEIJEIRAS, R., "La memoria de un rey victorioso: los sepul- cros de Alfonso VIII y la fiesta del triunfo de la Santa Cruz", en Grabkunst und Sepulkralkultur in Spanien und Portugal, BORNGÄSSER, B., KARGE, H., KLEIN, B. (eds.), Frankfurt am Main, 2006, 289-315, esp. 298-310.

${ }^{61}$ SÁNCHEZ AMEIJEIRAS, R., "El 'çementerio real' de Alfonso VIII en las Huelgas de Burgos", Cultura, poder y mecenazgo. Semata: Ciencias sociais e humanidades, 10 (1998), 77-109. 


\title{
TEXTO ORIGINAL
}

\author{
War memorials: remembering and forgetting at Las Huelgas and beyond
}

Rose Walker

The Courtauld Institute of Art

Most of literature on war memorials has been written by those who study the early modern and modern periods and not by medievalists. ${ }^{1}$ The material is all too rich. It ranges from vast cemeteries to small village memorials, regardless of who won or lost. At a national level, monuments like The Cenotaph in London or the Arc de Triomphe in Paris are the focus of annual commemoration ceremonies. Finely balanced between glorification and pacificism, those acts remain immensely poignant. One aspect of these monuments is their emphasis on the regular soldier. In 1920 the Tomb of the Unknown Warrior was installed in Westminster Abbey to commemorate 'the many multitudes' that were killed in the First World War. Whilst the names of over five hundred French Generals are inscribed on the inside walls of the Arc de Triomphe in Paris, from 1920 it has also sheltered a Tomb of the Unknown Soldier. In Germany, it was only in 1931 that a 'Memorial for the Fallen of the War' was created from the Neue Wache, but it became the focus for ideas of Nazi resurgence and so has now been rededicated as the 'Central Memorial of the Federal Republic of Germany for the Victims of War and Tyranny.' Many of these monuments are cumulative and record dead from the nineteenth century onwards, and tactily those that are to come. These monuments and their makers cannot control the memories that they evoke. Mary Carruthers has drawn attention to the ways in which the Vietnam Veterans' Memorial in Washington D.C. both connects with an established social tradition but also engenders particular responses. It has great social resonance in its form, 'the polished black granite, the individual naming of the dead' and in the site itself, yet it also requires visitors to tell their particular stories within the wider context of the memorial. In turn these stories become commentaries on the text of the Wall through a range of memorial actions, photographs, the rubbing of names or symbolic gifts. ${ }^{2}$ This kind of participatory remembering is typical of our time. But is the idea of war memorials completely anachronistic before the nineteenth century? Is there any physical evidence for recognition of the price of war in the Middle Ages?

The practice has been traced back to the end of the eighteenth century, to the period after the French Revolution. ${ }^{3}$ Those early war memorials have not survived, but accounts say that they were raised by Jacobins, supporters of the French Revolution. They commemorated groups and related to confraternities, and to that extent it may be possible to find some medieval precedents for their approach. Victory monuments had existed from the ancient world, most visibly now as Roman triumphal columns or arches, but it is much harder to find physical traces of concern for those who died in wars, especially for the regular soldiers. The ara pacis of the Emperor Augustus offered a new vision of a peaceful and fecund state, and thereby perhaps enshrined the healing of a civil war, but the memories of the conflict are only present through their absence. The ara pacis may have been more concerned with forgetting. In the Middle Ages it is even harder to identify war memorials, because victory and defeat were so inextricably linked to God. ${ }^{4}$ In the ideology of that time, God gave victory in accordance with just deserts. A great victory could secure a throne, and a defeat lose one. Yet the matter could be more complex, especially when the victory was that of one Christian king over another. Twelfth-century sources depicted William the Conqueror foundation of Battle Abbey on the site of the battle of Hastings in 1070 as not only a memorial for the victory that God had given him but also as an atonement especially for "those who fall here". ${ }^{5}$ The high altar was to mark the place where King Harald had been killed. Yet, as Elizabeth Hallam has said, "the likelihood is that the king himself viewed them as symbols of power rather more than as gestures of contrition."

After his successful siege of the city of Santarem in 1147, Afonso I of Portugal brought Cistercians to found Alcobaça (c.1153), which became an important focus for that new kingdom but not explicitly a war memorial. ${ }^{6}$ 
Another more overt victory monument from the twelfth century is the El-Hassan mosque in Salé (Rabat). The city was built between 1196 and 1197 to mark the great victory of the Almohad emir, Ya'qub, known as Miramamolín, over King Alfonso VIII of Castile at Alarcos. Alarcos was the momentous defeat of Alfonso VIII on 19 July 1195 , for which the emir took the title 'al-Mansur Billah' (Made Victorious by God). Various accounts suggest that the number of dead ranged from 30,000 Christians to 500 Muslims, or less plausibly 300,000 to 20,000. In any case, there is no doubt that the Christian dead vastly out-numbered the Muslims. According to Al-Himyari writing from earlier sources in the fifteenth century, the Almohads also took booty and captives who were put to work on the construction of the Mosque. ${ }^{7}$ The El Hassan mosque remained unfinished perhaps because Miramamolín died in 1199 , or because the victory was overwhelmingly reversed in 1212. Nonetheless, the tower and the large space mapped out for its hall and courtyard continued to dominate the site as an evocative reminder of the Almohad empire and the mosque that might have been.

These victory monuments seem to lack the qualities that are now associated with the term 'war memorial'. There is little indication of concern for the fallen, for the disasters of war or for the individual soldier. To appreciate that kind of medieval sensitivity, we have to turn to defeat, and a well-documented case is the inverse view of Alarcos, from the standpoint of King Alfonso VIII of Castile. The Latin Chronicle provides the most detailed account of the defeat at Alarcos. ${ }^{8}$ Alfonso VIII had seriously underestimated the Berber forces and decided not wait for the King of León to arrive before engaging them in battle. ${ }^{9}$ His own forces were tired and in some disorder as he had already offered battle on the preceding day, which had been refused by the emir. The Chronicle summarised the result: "A day prodigal of human blood sent Moors to hell and Christians to eternal mansions." 10 It recounts how the king tried to rush forward, but that his men 'besought him to retreat and save his life, because the Lord God seemed to be angry with the Christian people'. They led him away unwillingly, 'lamenting and bemoaning the great disaster that had happened'. The Order of Santiago lost nineteen of its members, and the majority of the Order of Calatrava was slain on the battlefield. ${ }^{11}$ The bishops of Ávila, Segovia and Sigüenza were amongst the dead as were some of Alfonso VIII's closest friends or supporters including Pedro Rodriguez de Guzmán, his mayordomo, with his son-in-law Rodrigo Sánchez, and Ordoño García de Roa and his brothers. ${ }^{12}$ This was a personal as well as a political catastrophe. The number of the dead meant that this was, as Joseph O'Callaghan has stated, "the worst defeat suffered by any Christian king since Alfonso VI's rout at Zallaqa a century before". ${ }^{13}$

The wider world did not fail to notice the extent of the disaster. The troubadour Folquet de Marseilles lamented the profound fall (profunda caída) of Alarcos (Hueimas no y conosc razo). ${ }^{14}$ The wider context provided by the song is the loss of Jerusalem by the Christians in 1187: ' we already lost the Sepulchre' (Qu' el sepulcre perdem premeiramen). Alfonso VIII is implicitly blamed for proceeding without God on his side, as 'all effort without God is as nothing', although there is no more specific criticism of the king. To allow such disasters God must be angry with Christendom. Folquet's song is also a call to arms and, unsurprisingly given his location in the south of France, he urges the inclusion of the King of Aragón in the fightback. This was not an unbiased view. In England, the thirteenth-century English Chronica majora of Matthew Paris records under the year 1195 that 'all Christendom, thrown into confusion, was shaken to the core'. ${ }^{15}$ In retrospect, after the victory of Las Navas de Tolosa in 1212, even the Archbishop of Toledo, Rodrigo Jímenez de Rada, the great chronicler, allows himself to refer to 'the dishonour for some time borne' (oprobrium aliquandiu toleratum) in the presence of the king. ${ }^{16}$

The sources suggest that Alfonso VIII responded to the defeat in a variety of ways. Politically, the internal disputes between León, Castile, Navarre and Aragón continued, but Alfonso VIII also began to build alliances especially through the marriages of his daughters, Berenguela to the King of León in 1197, Urraca to the King of Portugal and Blanca to the son of the King of France in 1200. The Latin Chronicle also describes Alfonso's pleasure in the accomplishments of his eldest son, Fernando. But alongside this, 'the misfortune which he had suffered in the battle of Alarcos remained fixed deep in the king's mind; he had never erased it. Oftentimes he recalled that day to mind, desiring to have revenge on the king of Morocco, and for this he often prayed to the Lord. ${ }^{17}$ Alfonso VIII had also made reparation to the Order of Calatrava, and in the charter that gave property in Ronda stated that he was "sorrowing for your poverty, the loss of your greater home of Calatrava and of almost all your possessions because of the misfortune of Alarcos." 18

The king was to suffer more painful reverses before the final victory of Las Navas de Tolosa, especially in 1211. Unable to assemble a sufficiently large army, he had to surrender the castle of Salvtierra in September 1211, albeit without loss of life. The Latin Chronicle expresses the reaction in biblical language, 'O what great mourning among men, the cry of women moaning and striking their breasts for the loss of Salvatierra!'. ${ }^{19}$ In October the same year Alfonso's son, Fernando, died of a fever at Madrid, and again the Chronicle turns to biblical texts, including Jeremiah and Lamentations, to try to express the extreme grief of Alfonso VIII and his queen Leonor. 
This tragic death brought Alfonso VIII of Castile the same double calamity that had befallen Alfonso VI of León and Castile. Alfonso VI had lost the battle of Zallaqa (Sagrajas) to the Almoravids in 1086, ${ }^{20}$ and his son and heir, Sancho, in an engagement at Uclés in 1108.

This comparison may help to demonstrate the depth of emotional response to such a terrible sequence of tragedies. The events that contributed to Alfonso VI's nadir are all included in the crónica najerense, an anonymous compilation, probably written at the abbey of Santa María de Nájera after $1173 .{ }^{21}$ It was to be an important source for the major chronicles of the thirteenth century, the De rebus hispanie of Archbishop Rodrigo Jiménez de Rada and the Chronicon mundi of Lucas de Tuy. It contains the briefest of references to Zallaqa, but gives the precise date of the death of Sancho at Uclés, on the day of the nativity of St John the Baptist in $1108,{ }^{22}$ and says that he was killed with his military tutor, García Ordoñez and six other counts. Garsía Ordoñez was probably buried at Santa María de Nájera. ${ }^{23}$ The next and last chapter of the crónica najerense (III.23), after a passage glorifying Alfonso VI, devotes most of the remaining lines to a description of a great miracle (magnum fecit miraculum Deus omnipotens) at San Isidoro de León on the Octave before Alfonso Vl's death. This takes place on the day of the Nativity of St John the Baptist, that is on the anniversary of the battle of Uclés and the death of his son. Water poured from the stones before the altar of St Isidore, not between the stones but out of them (non per iuncturas set per medium petrarum) for four days. The flow ceased only after a procession from the cathedral, the performance of mass and after some of the water had been drunk and more collected in a glass vessel. The chronicler says that the stones wept such that they moved men to tears (Ideoque plorauerunt lapides ut ad fletum homines commouerent) and that this sign was for the mourning and tribulations that came to Spain after the death of Alfonso VI (Hoc signum nichil aliud portendebat nisi luctus et tribulationes que Yspanie post eius obitum euenerunt.) But the literary construction of the text and the emphasis given to the anniversary and the sequence of tragic events, it is difficult not to see the miracle story also as weeping for the double tragedy at the end of the king's otherwise highly successful life, the defeats at al-Zallaqa and Uclés and above all the death of his heir Sancho.

The castle at Uclés was given to Alfonso VII as part of a treaty with Lobo de Múrcia. Alfonso VII gave it to his son Sancho III, Alfonso VIII's father. In 1163, when Alfonso VIII was still a minor, he, together with his uncle Fernando II of León, gave the castle to the military Order of St. John and to the new Order of Santiago. ${ }^{24}$ In 1172 , around the time that the chronica najerense was written, Alfonso VIII augmented the Order of Santiago with the friars of Ávila. The agreement was full of optimism and asserted that as soon as the Muslims had been expelled from Spain, the friars would advance on Jerusalem by way of Morocco. ${ }^{25}$ By 1174 the castle of Uclés had become the headquarters of the Order (Caput Ordinis), with a convent church dedicated to the Virgin, the protectress of the reconquista. It is difficult to know the extent to which unfortunate family memories attached to this site. Whether the decision to give it to the Order of Santiago was a purely strategic one or also designed to purge the past and reclaim the landscape can be only the subject of speculation.

Thus I think we can be confident that the emotions necessary for a 'war memorial' existed in the kingdom of Castile after the defeat of Alarcos. But would it have been appropriate to memorialise a defeat in that society? If the dead were honoured, it might be easier to assemble a great army in the future. Perhaps Castile needed to be healed. It certainly needed to find a way of ensuring that God was on its side. In medieval parlance, Castile needed to undergo penance in the person of its king. In some of the literary and historical interstices of the texts known as the Primera Crónica General or the Estoria de España, written at the end of the thirteenth and in the fourteenth centuries, Alfonso VIII is represented as a sinner. His fault is an affair with a Jewish woman, Fermosa, for which he was punished at Alarcos. The first mention was in a margin of the 1289 manuscript but the story grew with the telling. One element of the legend says that Alfonso VIII built the monastery of Santa María Regalis de Las Huelgas as an act of repentance. ${ }^{26}$ As there is no contemporary reference to this affair in the songs of the troubadours or in the chronicle of De Rada, who was close to the family, it is often discounted. However, although the affair with a Jewish woman may remain doubtful, the idea of penance may indeed have been crucial in the development of the monastery of Las Huelgas. It is possible that the legend of Fermosa reinvented a more difficult reality, Alfonso VIII's initial lack of military prowess. De Rada, on the other hand, links the defeat at Alarcos to the foundation of Las Huelgas only by juxtaposition. The sentence that comes before the description of the foundation of Las Huelgas says that Alfonso kept the battle of Alarcos 'very high in his heart'. ${ }^{27}$ Las Huelgas was the primary foundation of Alfonso VIII and his queen Leonor. It was set up in 1187 on the outskirts of Burgos and held its first Annual Chapter in $1189 .{ }^{28}$ In short, the conception of Las Huelgas preceded Alarcos by several years. However, its first cloister, known as Las Claustrillas, may well belong to the late 1190 s..$^{29}$ I have previously suggested that this cloister was built under the shadow of the loss of Jerusalem in 1187, that its form may have reflected its role as a burial site for not only the infante Fernando but also for one or more of the dead from 
Alarcos. ${ }^{30}$ I now wish to explore the third idea in more detail, that Las Claustrillas was at least partly a response to Alfonso VIII's guilt.

Further documentary evidence connects Las Huelgas to Alarcos, in the form of one of the nobles named in the Latin Chronicle as having died on the battlefield of Alarcos, Pedro Rodríguez de Guzmán. ${ }^{31}$ He, and possibly Ordoño García who also died at Alarcos, was one of the signatories to the 1187 foundation charter of Las Huelgas. ${ }^{32}$ In 1194, Pedro Rodríguez de Guzmán and his wife Mafalda gave property to Las Huelgas 'pro Dei amore et animarum nostrarum et parentum nostrum redemptione', property in Revilla del Campo, in both Hontorias and in Quintanaseca, that was to be handed over on the death of whichever of them died first. ${ }^{33}$ Four years after the death of Pedro Rodríguez, in 1199, when Las Huelgas fully realised its Cistercian affiliation, two of its nuns, daughters of Pedro Rodríguez, made another donation to Las Huelgas. They gave, in accordance with their father's will, all their inheritance together with their bodies and everything that they had from their father to the abbess of Las Huelgas pro anima for their father and themselves. ${ }^{34}$ It is possible that one of the sisters was the widow of the named son-in-law of Pedro Rodríguez, Rodrigo Sánchez. Their brothers, Nuño Pérez and Guillem Pérez fought at Las Navas together with the powerful Thibaut de Blazón of Poitou, who, according to the Latin Chronicle was also the son of Pedro Rodríguez. ${ }^{35}$ This might explain why he, and the Catalonian archbishop of Narbonne, were the only leaders of ultramontane forces to stay the course and join the Spanish kings at Las Navas. ${ }^{36}$ The daughters of Pedro Rodríguez were successful in the abbey, and Teresa, presumably the elder, became cantatrix by 1211, and, in 1232, María became abbess. In 1234, María made another major donation to Las Huelgas for her and her sister's souls, with the imprimatur of King Fernando III. ${ }^{37}$ Thus the memory of Alarcos may have been particularly strong at Las Huelgas, if it was kept alive by the daughters of Pedro Rodríguez. Their father may even have been celebrated as a martyr. There is no specific evidence that the daughters's donations were used to fund the building of the cloister, but they joined the abbey at the right time for that to be the case. The sculptural decoration in Las Claustrillas is unusually eclectic and dissonant, and one explanation for that could be the involvement of a number of families in the sculptural decoration in Las Claustrillas. However, such arguments are at best circumstancial.

If part of the abbey of Las Huelgas was built as a penance for Alarcos and the community of nuns had specific links to its memory, is it possible to discern that more concretely in the fabric? The most distinctive decoration in Las Claustrillas is applied to the intermediate pier of the north arcade. (fig. 1) This represents the templum domini, and perhaps marks the whole cloister as the Tabernacle. ${ }^{38}$ I have previously seen these pierced intermediate piers as primarily connected to the funerary function of the cloister, and I continue to believe that that is one level of meaning. However, I think that more weight should perhaps be given to my secondary suggestion that the representations of the Temple/Tabernacle may relate to penitential activity, as defined by Mary Carruthers. ${ }^{39}$ She has analysed the way in which the measuring of the Tabernacle described in chapter 43 of the Book of Ezechiel, where the Tabernacle is measured, became such a penitential activity.

Thou son of man, show the temple to the house of Israel, that they may be ashamed of their iniquities: let them measure the pattern, and blush from all they have done. Show them the plan of the house, and of its fabric, its exits and entrances, and its whole description, and all of its precepts, [and the rest of its ordering, and all its laws, and write [them] before their sight so that they may store away all its descriptions and its precepts, and fashion them]." (Ezechiel 43.10-11)

Carruthers maintains that this "measuring the pattern" of the Holy City became a trope of "penitential remembering" and a commonplace of monastic rhetoric. The biblical text is "the ritual of Israel's penitence and reconciliation with God", the goal of which is "the covenant of salvation". It thus provides a penance that operates at a national level and would have perfectly fitted Castile's need for such reconciliation with the Almighty. Biblical exegesis of the passage, for example that of Hrabanus Maurus, talks of this penitence as an activity: while we 'measure the fabric', we must blush in shame over all the things which we do." This sounds more personal and a suitable penance for Alfonso VIII's mismanagement of the campaign. Overall "measuring the pattern" was ideal penitential activity for a sin that was both personal and social. For Carruthers it is also "an act of contrition, routed through an architectural mnemonic schema." This memory work could be done with a two-dimensional image such as the plan of the Tabernacle in the Codex Amiatinus (Florence, Biblioteca Medicea Laurenziana MS Amiatinus I, ff IIv-III) where the mind's eye could move both behind and in front of the columns and curtains. ${ }^{40} 41$ But it could also include liturgy, the sacrament of penance, and more public activities like processions. The ductus of the memory work could roam around and through a three-dimensional space such as a monastic cloister, and particularly one like Las Claustrillas with its pierced piers, columns and sculpted curtains. ${ }^{42}$ Above all, this process 
of Biblical measurings had the ability to "reconcile an entire community with God." ${ }^{43}$ After the defeat at Alarcos, Alfonso VIII had great need of this activity, on his own behalf and on behalf of the kingdom of Castile. However appropriate this activity may have been, there needs to be evidence that it took place at Las Huelgas. The Primera Crónica de España may provide the first piece of that evidence. It says that the holy consecrated virgins, 'enclosed in the cloister and the church', were 'to praise and sing to God day and night' to ask his favour for his Christian people 'so that he might protect and direct them correctly (enderesce) to his service and to his right side (diestra parte)' ${ }^{44}$ The verb enderesce has connotations of straightening and rectifying, and the emphasis on God's right side also implies correction. The liturgy of the nuns is thus credited with the ability to intercede for what the armies of Castile needed, the favour of God.

The sculptural decoration of the cloister of Las Claustrillas provides further evidence, as a concrete expression of that penitential activity. The whole cloister may be metaphorically the Tabernacle, and there may even be some number symbolism in the six bays either side of the intermediate piers at Las Claustrillas, as the number six plays such an important part in the measurements of the Temple in Ezechiel. Most importantly the intermediate pier in the north gallery evokes the Temple. On its south face, four orders of columns surround the opening in the pier and sculpted curtains wind around them. Above symmetrical micro-architectural decoration extends into overhanging corbels. On the north face, the garth side, the columns and curtains seem unfinished or damaged but the micro-architecture is much more varied but also extends into overhanging corbels. (fig. 2) This identification is strengthened by a comparison with an image in the Cardeña Beatus (Madrid, Museo Arqueológico Nacional, MS 2, fol.106). (fig. 3) The Cardeña Beatus is an undated manuscript usually placed around 1180 on stylistic grounds, but the close and unusual nature of this comparison could suggest that it might be better placed shortly after the fall of Jerusalem in 1187 and perhaps c. $1190 .{ }^{45}$ The rubric on this folio says that it shows Antichristus overthrowing the city of Jerusalem and the killing of the two witnesses, Enoch and Elias. These scenes are depicted in two registers with the destruction of Jerusalem in the upper register. Soldiers attack, and indeed are beginning to demolish Jerusalem, which is represented as a large arch flanked by two columns that shelters the population. Above the arch micro-architectural decoration extends into two towers built on overhanging corbels. There are sufficient similarities to suggest that the images were created in the same milieu, and indeed it has been suggested that the Cardeña Beatus was produced near Burgos, at the monastery of San Pedro de Cardeña perhaps even with the involvement of Alfonso VIII and Leonor. ${ }^{46}$ The Rylands Beatus (Manchester, John Rylands University Library, MS lat. 8, fol.134v) uses a similar architectural framework to illustrate the passage in the Book of Revelation where John measures the Temple of God with a rod. ${ }^{47}$ Therefore I am proposing that Las Claustrillas was designed in this rich liturgical and political context, the overthrow of Jerusalem and the defeat of Alarcos. It did not merely reflect it, but was to be an active part, through liturgical performance and memory work. Through contemplation of the ways around and through the cloister, and by means of constant praise and prayer, Las Huelgas was to be the spiritual powerhouse that would lead to the victory of Las Navas de Tolosa. As Cantatrix, Teresa Pérez may have overseen much of this intensive musical and liturgical work. The pentitential activity could have provided not only a supportive environment but also by drawing the memories into the architectural space it could have freed Alfonso VIII and his advisors to concentrate on the analysis of the military and political errors that had led to the defeat at Alarcos, and on avoiding those problems after the end of the treaty with the Almohads. ${ }^{48}$ As those working on memory and buildings in the early modern and modern periods have suggested architecture can be more an instrument of forgetting than of memory, as it can stand in for the work of remembering ${ }^{49}$ Las Claustrillas may provide a medieval example of that process.

But does this enable us to say that Las Claustrillas was in some sense a war memorial? I think there is sufficient evidence to answer in the affirmative. Las Claustrillas was unusual in that it memorialised a defeat, and that it was, therefore, dynamic, working towards its own obsolescence. However, in one important way it differed from the war memorials of the nineteenth and twentieth centuries, it was in a private and not a public space. There was interaction and performance, but mainly for the nuns and chaplains of the abbey, and perhaps for Alfonso VIII, Queen Leonor and other members of the royal family. There may have been commemoration of one or more martyrs of Alarcos, for Pedro Rodríguez and his son-in-law Rodrigo Sánchez, but if so, it was a private matter. Was there a memorial for the multitude of dead and was there one to which everyone could have access? There are several possible answers to those questions but in nearly every case they relate to the Military Orders.

There is certainly awareness of the contribution of the soldiers, and it is expressed clearly by Rodrigo Jiménez de Rada in his De rebus hispanie immediately after the victory of Las Navas. He puts into his own mouth a reminder to Alfonso VIII: 'be mindful of your soldiers, through whose help you have come to such great glory'. ${ }^{50}$ Regular soldiers may also be depicted on a capital from the monastery of San Pedro de Gumiel de Izán (Burgos). 
(fig. 4) San Pedro de Gumiel was the monastery where Diego Velásquez, one of the early members of the Order of Calatrava, returned after Alarcos, and he was buried there in 1196. De Rada seems to remember him with respect and perhaps fondness. ${ }^{51}$ His body was the focus of a cult in later years. ${ }^{52}$ San Pedro de Gumiel was a place of commemoration for the Order of Calatrava after the defeat. There are clear stylistic links between the foliate work on one of the corner piers at Las Claustrillas and sculpture from San Pedro de Gumiel. ${ }^{53}$ Yet Gumiel has other sculpture that is very different from anything at Las Claustrillas, indeed from any art that has been defined as 'Crusader Art'. ${ }^{54}$ Most notably disembodied heads of men protrude starkly from the core of one capital. Some wear flat protective caps, others more elaborate helmets that cover the lower part of their faces. This composition is very different from the more popular representations of victorious knights. ${ }^{55}$ They seem to evoke a brotherhood and its demise with a simplicity that is more often associated with art of the twentieth century. Other memorial sites for the Order were in Guadalajara at Zorita de los Canes and Alcañiz, and perhaps most problematically at Montsalud, where Nuño Perez de Quiñones, the Master of the Order who had failed in his in command at Alarcos, was buried. ${ }^{56}$ Thus after Alarcos the memory of the fallen from the Order of Calatrava was dispersed, like the body parts of so many of its members.

The field of Alarcos was probably for a time the only sepulchre for the unnamed dead, despite a much later suggestion that a chapel was erected for them at Alarcos dedicated to Nuestra Señora de los Martires. ${ }^{57}$ As Victor Hugo said of the field of Waterloo, 'There is no French tomb. The whole of that plain is a sepulchre for France.' Ultimately their memory was to be gathered together with that of the smaller number who had died at Las Navas. It was to be concentrated on the pass protected by Salvatierra, the road to Andalucia. (fig. 5) This whole landscape was thus deeply imbued with the memories of the campaigns and their dead. And it was the landscape that remained the public 'war memorial'. The pass is still a striking piece of topography guarded by the ruined castle of Salvatierra to the east and the more substantial remains of Calatrava la Nueva to the west. Salvatierra was the castle from which the soldiers of the Order of Calatrava had sallied forth to fight and die at Alarcos. Its loss had been the major symbolic event of the annus horribilis of 1211. It was no longer 'the castle of salvation' but, in the words of the fourteenth-century Muslim historian Ibn Idhari, 'the place of expiation of their sins'. Although Salvatierra was reoccupied, it was no longer to enshrine the identity and memory of the Order of Calatrava. (fig. 6) From 1217 the site of this memory was the new castle, Calatrava la Nueva, on the opposite side of the pass from Salvatierra. ${ }^{58}$ The remains of the dead both from Alarcos and from Las Navas de Tolosa were gathering up and translated together to the new site. Their presence in a cemetery constructed within a claustral platform sacralized the new site. Both groups were to be honoured as martyrs within one monument. A chapel was dedicated to the Virgin, as Nuestra Señora de los Mártires, and many Masters of the Order were later to be buried on that site 'ad sanctos' ${ }^{59}$ (fig. 7) The Virgin was thus to preside over the memory and intercession of the members of the Order whether martyred at Alarcos or Las Navas de Tolosa. I think it is possible to say that the chapel in Calatrava la Nueva was a war memorial for the Order, for a confraternity, and that it continued to function as such and to embody those memories as a crucial part of its group identity for centuries.

In Burgos at Las Huelgas the painful memories of Alarcos were likewise resolved and subsumed. The penitential activity had apparently worked. Under the protection of the Virgin as Santa María Regalis, Las Huelgas metamorphosed into a repository for the memory of the victory at Las Navas de Tolosa. As Rocío Sánchez has shown so definitively, the event was celebrated at Las Huelgas on the Feast of the Exaltation of the Holy Cross. The liturgy even incorporated sections of De Rada's De rebus hispanie. ${ }^{60}$ Alfonso VIII had become the victorious king, feted across Christendom, even if he was to enjoy it for only a couple of years before his death in 1214. Las Huelgas was to be reinvented as a Gothic Cistercian monastery, and eventually to dominate its late Romanesque cloister of Las Claustrillas with a new Gothic claustral space. And within the confines of the old cloister that carried so many memories the small Chapel of the Assumption, where the bodies of Alfonso VIII and Leonor may have originally rested, was to receive new decorative vaulting that appropriated the finest stuccowork of their Almohad defeated enemies. ${ }^{61}$ Memorialisation had reverted to the more traditional celebration of victory. 


\section{NOTES}

1 I wish to thank Tom Nickson for giving me the opportunity to present this as a paper at the conference on 'Constructing Memory in Medieval Spain' at the University of York in 2011.

2 CARRUTHERS, M., The Craft of Thought. Meditation, Rhetoric, and the Making of Images, 400-1200, Cambridge, 1998, 35-40. See also ROWLANDS, M., "Remembering to Forget: Sublimation as Sacrifice in War Memorials", in FORTY, A. and KÜCHLER, S., The Art of Forgetting, Oxford, 1999, 129-146.

${ }^{3}$ CLARKE, J., "The Sacred Names of the Nation's Dead: War and Remembrance in Revolutionary France", in Memory, Mourning, Landscape, ANDERSON, E., MADDRELL, A., MCLOUGHLIN, K., VINCENT, A., (eds.), Amsterdam and New York, 2010, 21-42.

${ }^{4}$ For a survey of war memorials, BORG, A., War memorials: from antiquity to the present, London, 1991 and ibid. "Some Medieval War Memorials", in Medieval Architecture and its intellectual context, studies in honour of Peter Kidson, FERNIE, E. and CROSSLEY, P. (eds.), London, 1990, 1-7.

${ }^{5}$ HALLAM, E.M., "Monasteries as 'War Memorials': Battle Abbey and La Victoire," in The Church and War, Studies in Church History, 20, 1983, 47-57; esp.53; FERNIE, E., The Architecture of Norman England, Oxford, 2000, 10203.

6 JANAUSCHEK, P.L., Originum Cisterciensium, vol. 1, Vindobonae, 1877, 110.

7 SMITH, C., Christians and Moors in Spain, vol.3. Arabic Sources (7111501), Warminster, 1992, 134-137.

8 Chronica latina regum Castellae, CHARLO BREA, L., ESTÉVEZ SOLA, J.A., and CARANDE HERRERO, R. (eds.) Chronica hispana saeculi XIII. Corpus christianorum. Continuatio medievalis 73, Turnholt, 1997, 44-47, ch.12-13; The Latin Chronicle of the Kings of Castile, O'CALLAGHAN, J. (trans.). Tempe, 2002, 26-27.

9 VANN, T. M., "Battle of Alarcos", in Medieval Iberia. An encyclopaedia, GERLI, E. M. (ed.), New York, 2003,
42-3. O'CALLAGHAN, J. F., Reconquest and Crusade in Medieval Spain, Philadelphia, 2003, 61.

10 The Latin Chronicle, 26.

11 LOMAX, D., La Orden de Santiago (1170-1275), Madrid, 1965, 90; O'CALLAGHAN, J.F., "The Order of Calatrava: Years of Crisis and Survival, 1158-1212," in The Meeting of Two Worlds: Cultural Exchange Between East and West During the Period of the Crusades, Kalamazoo, 1986, 419-430.

12 ANIZ IRIATE, C. and DÍAZ MARTíN, L.V., Santo Domingo de Caleruega en su context socio-político, 11701221. Jornadas de Estudios Medievales, Salamanca, 1994, 199. The death of Pedro Rodríguez de Guzmán on 18 July 1195 was also noted in the obituary of Burgos Cathedral, SERRANO, L., El obispado de Burgos y Castilla primitiva desde el siglo $V$ al XIII, Madrid, 1935, 385.

13 O'CALLAGHAN, Reconquest, 61-2.

${ }^{14}$ MILÁ y FONTANELS, M., De los trovadores en España: Estudio de lengua y poesía provenzal, Barcelona, 1861, 114-116.

15 MATTHEW PARIS, Chronica majora, Rerum britannicarum medii evi scriptores 57, vol.2, 1067-1216, LUARD, H.R, (ed.), London, 1872-83, 410: De horribili adventu paganorum in Hispaniam... 'tota Christianitas contremuit conturbata.'

16 RODRIGO JÍMENEZ DE RADA, Historia de rebus Hispanie sive Historia Gothica, FERNÁNDEZ VALVERDE, J. (ed.), Corpus Christianorum. Continuatio Mediaevalis 72, Turnhout, 1987, 8.10: "Estote memor gracie Dei, que omnes deffectus in uobis suppleuit et oprobrium aliquandiu toleratum hodie releuauit."

17 The Latin Chronicle, 36-37.

18 GONZÁlEZ GONZÁLEZ, J., El reino de Castilla en la época de Alfonso VIII, Madrid, 1960, 2, 164-66, doc. 658: "condolens paupertate eo quod domum uestrum maiorem de Calatraua uestrasque omnes fere res ex infortunio de Alarcos"; RADES Y ANDRADA, F., Crónica de las tres Órdenes de Santiago, Calatrava y Alcántara (facsimile con un studio sobre "La obra historica de Rades y Andrada", by LOMAX, D.W., Biblioteca de Historia Hispanica, Órdenes Militares, Serie Maior 2, Barcelona, 1980.

19 The Latin Chronicle, 39.

20 SMITH, C., Christians and Moors in Spain, vol.3. Arabic Sources (711-1501), Warminster, 1992, 92-99.

21 ESTÉVEZ SOLA, J., Chronica Hispana saeculi XII, Pars II: Chronica Naierensis, Corpus Christianorum, Continuatio Medievalis, LXXI, Turnhout, 1995

22 Ibid., 3. 21-22: 'ubi etiam occisus est comes Garsias de Grannione cognomento Crispus et sex alii comites cum eo'.

23 REGLERO DE LA FUENTE, C. M., "La Crónica najerense, Santa María de Nájera y Cluny", e-Spania [Online], 7 June 2009, Online since 01 July 2009, connection on 26 August 2012. URL : http://e-spania.revues.org/18162; DOI : 10.4000/e-spania.18162, para. 63.

24 RIVERA GARRETAS, M., 'La orden de Santiago en Castilla la Nueva en los siglos XII y XIII', in Los Órdenes Militares en el Mediterráneo occidental: Siglos XIII-XVIII: coloquio celebrado los días 4, 5 y 6 de mayo de 1983, Madrid, 1989, 23-40.

${ }^{25}$ O'CALLAGHAN, Reconquest, 5.

26 DYER, N.J., "Alfonsine Historiography: The Literary Narrative", in Emperor of Culture. Alfonso $X$ the Learned of Castile and His ThirteenthCentury Renaissance, BURNS, R.I., (ed.), Philadelphia, 1990, 141-158; DEYERMOND, A.D., A Literary History of Spain. The Middle Ages, London and New York, 1971, 88-89; MENÉNDEZ PIDAL, R., Primera Crónica de España, 2, Madrid, 1977, 685 ch.1006: "El muy noble rey da Alffonsso tiene condesada en el muy alto su coraçon la batalla de Alarcos, et en lo que guisarlo pudiese, querisse uengar, como prinçep de grand poder et de muy grand coraçon, en qualquiera manera et en qualquiera logar que lo el guisar pudiesse, de lo que fuera maltrecho en a quella batalla de Alarcos ... començo a laurer et a fazar çerca Burgos un monesterio de duennas de la orden de Çesteles."

27 DE RADA, De rebus, 7.33. 
28 WALKER, R., "Leonor of England, Plantagenet queen of King Alfonso VIII of Castile, and her foundation of the Cistercian abbey of Las Huelgas. In imitation of Fontevraud?", Journal of Medieval History, 31 (2005) 346-368;

${ }^{29}$ WALKER, R., "The Poetics of Defeat: Cistercians and Frontier Gothic at the Abbey of Las Huelgas", in HOURIHANE, C., (ed.), Spanish Medieval Art: Recent Studies, Tempe, 2007, 187-213, esp. 189-195.

30 ibid., 212-213.

31 The Latin Chronicle, 26 (chapter 13): "In the first rank of the Christians great men fell: Ordoñõ García de Roda and his brothers, Pedro Rodríguez de Guzmán, Rodrigo Sánchez, his sonin-law, and many others."

32 LIZOAIN GARRIDO, J. M., Documentación del monasterio de las Huelgas de Burgos (1116-1230), vol.1, Burgos, 1985-90, no.11, 19-23.

33 LIZOAIN GARRIDO, Documentación, no. 36, 66-67. Also property called Riofocin to their daughters Teresa and María that after their deaths would revert to their brothers or their descendents.

${ }^{34}$ LIZOAIN GARRIDO, Documentación, no.50, 87-89: 'pro divisione quod fecit pater noster, dompnus Potro Roderici de Guzman, pro anima eius et pro animabus nostris donamus [... to the abbess Maria Gutierrez and her successors ...] totam illam nostram hereditatem, quantum habemus et habere in Ribilla del Campo et in Fontorias, cum quantum nobis ibi pertinet ex parte patre nostro Petro Roderici de Guzman ... cum corporibus nostris iure heditario in perpetuum", confirmed by the infans Fredinandus, the queen's mayordomo, and their brother Nuño Pérez. See also ANIZ IRIATE, Santo Domingo, 199-201.

35 GÓMEZ, M.D., The Battle of Las Navas de Tolosa: the Culture and Practice of Crusading in Medieval Iberia, Unpubl. PhD. Dissertation, University of Tennesse-Knoxville, 2011, 124-126. DE RADA, (8.6.50-2), confirms that he was "a noble and energetic man, Spanish by birth and Castilian by family ("homo nobilis et strenuus et natione Hispanus et genere (astellanus"). It is somewhat puzzling that Thibaut should have been both the Spanish son of Pedro Rodríguez and lord of Blazón. One possibility is that the connection was through his mother Mafalda. Pedro Rodríguez and Mafalda were married in 1174, when Alfonso VIII gave Mafalda a wedding gift (in casamento), see GONZÁLEZ, El reino, 2, 338-339, doc.205.

36 O'CALLAGHAN, The Latin Chronicle, ch. 22, 45-46: "a few remained with Theobald of Blazón, the son of Pedro Rodríguez de Guzmán, and with the archbishop of Narbonne who had been born in Catalonia."

37 LIZOAIN GARRIDO, Documentación: no.106, 170-171, where Teresa Peidrez, cantatrix confirms after the prioress; ibid. Documentación, vol.3, 1263-1283, no. 276, 40-41, "donna Maria Perez de Guzman, por la gracia de Dios abbadessa del monesterio de Sancta Maria la Real de Burgos, do por mi alma e de donna Teresa, mi hermana"

38 WALKER, "The Poetics", 210211.

39 Ibid., 213. CARRUTHERS, 231237.

40 CARRUTHERS, 234-237 and pl.24

41 REVEL-NEHER, E., "Du Codex Amiatinus et ses rapports avec les plans du Tabernacle dans l'art juif et dans l'art byzantine," The Journal of Jewish Art, 9 (1982) 6-17.

${ }^{42}$ CARRUTHERS, The Craft, 27276.

${ }^{43}$ Ibid. The Craft, 233.

${ }_{44}$ MENÉNDEZ PIDAL, Primera Crónica, 2, ".. porque las sanctas virgenes conssagradas, que alabassen et cantassen alli a Dios de dia et de noche non sintiessen ninguna mingua de todas casas ouiessen mester: mas que, ençerradas alli en la claustra et en la eglesia et en las otras moradas ... en cantar a Dios et alabarle et pedirle merced por su pueblo cristiano que le ampare yl enderesce al su servitio y que a su diestra parte."

45 WILLIAMS, J., The Illustrated Beatus. A Corpus of the lllustrations of the Commentary on the Apocalypse, vol. V, The Twelfth and Thirteenth Centuries, London, 2003, 24-30 and fig. 168.
46 Ibid., 24-27. WALKER, R., "Leonor of England and Eleanor of Castile: Anglo-lberian marriage and cultural exchange in the twelfth and thirteenth centuries", in BULLÓN-FERNÁNDEZ, M. (ed.), England and Iberia in the Middle Ages, 12th-15th century. Cultural, literary, and political exchanges, New York, 2007, 72-73.

47 WILLIAMS, The Illustrated Beatus, 19-23, and fig. 68.

48 ALVIRA CABRER, M., "De Alarcos a las Navas de Tolosa: Idea y realidad de los orígenes de la batalla de 1212", in in Actas del Congreso Internacional Conmemorativo del VII Centenario de la Batalla de Alarcos, IZQUIERDO BENITO, R. and RUÍZ GÓMEZ, F. (eds.), Ciudad Real, 1996, 249-264, esp. 254.

49 FORTY, A. and KÜCHLER, S., The Art of Forgetting, Oxford, 1999.

50 DE RADA, 8.10.49: "Toletanus pontifex hec uerba dixit nobili regi: ".... Estote etiam memor uestrorum militum, quorum auxilio ad tantam gloriam peruenistis".

${ }^{51}$ Ibid., 7.14: "Didacus Velasqui postea diu vixit, quem etiam memini me vidisse, et obit in Monasterio Sancti Petri de Gumiello, et ibi sepultus est, requiescat in pace."

52 MATEO, C., "Fray Diego de Velásquez (1196-1996)", Cistercium, Historia, Arte, Espiritualidad. Revista monastica, 205 (1996) 323.

53 WALKER, "The Poetics", 198204; for Gumiel, PÉREZ CARMONA, J., Arquitectura y escultura románicas en la provincia de Burgos, Burgos, 1959, 265-66, fig. 288 and 289.

${ }^{54}$ See, for example, FOLDA, J., Crusader Art: the art of the Crusaders in the Holy Land, 1099-1291, Aldershot, 2008; Gómez, M.D., "The Crusades and church art in the era of Las Navas de Tolosa", Anuario de historia de la iglesia, 20 (2011) 237-60.

55 RUIZ MALDONADO, M., "El 'caballero victorioso' en la escultura romaánica española. Algunas consideraciones y nuevos ejemplos, Boletín Seminario de Estudios de Arte y Arqueología, 1979, 271-286.

${ }^{56}$ LAYNA SERRANO, F., La arquitectura románica en la provincia de Guadalajara, Guadalajara, 2001, 180183, 185-189. 
36 War memorials: remembering and forgetting at Las Huelgas and beyond

${ }^{57}$ ZAPATA ALARCÓN, J., "El culto a los mártires: Visión y símbolo del medievo a la contrarreforma. La construcción del relicario del Sacro Convento de Calatrava la Nueva", in Actas del Congreso Internacional Conmemorativo del VII Centenario de la Batalla de Alarcos, IZQUIERDO BENITO, R. and RUíZ GÓMEZ, F. (eds.), Ciudad Real, 1996, 615-23.
58 Ibid. 616

59 Ibid., 616.

60 SÁNCHEZ AMEIJEIRAS, R., "La memoria de un rey victorioso: los sepulcros de Alfonso VIII y la fiesta del triunfo de la Santa Cruz," in Grabkunst und Sepulkralkultur in Spanien und Portugal, BORNGÄSSER, B., KARGE, H., KLEIN, B., Frankfurt am Main, 2006, 289-315, esp. 298-310.
61 SÁNCHEZ AMEIJEIRAS, R., "El 'çementerio real' de Alfonso VIII en las Huelgas de Burgos." in Cultura, poder y mecenazgo. Semata: Ciencias sociais e humanidades, 10 (1998), ed. VIGO TRASANCOS, A., Santiago de Compostela, 1998, 77-109. 